\title{
QuANTUM STATES ON HARMONIC LATTICES
}

\author{
Norbert Schuch, J. Ignacio Cirac, and Michael M. Wolf \\ Max-Planck-Institut für Quantenoptik, Hans-Kopfermann-Str. 1, D-85748 Garching, Germany.
}

(Dated: January 23, 2012)

We investigate bosonic Gaussian quantum states on an infinite cubic lattice in arbitrary spatial dimensions. We derive general properties of such states as ground states of quadratic Hamiltonians for both critical and non-critical cases. Tight analytic relations between the decay of the interaction and the correlation functions are proven and the dependence of the correlation length on band gap and effective mass is derived. We show that properties of critical ground states depend on the gap of the point-symmetrized rather than on that of the original Hamiltonian. For critical systems with polynomially decaying interactions logarithmic deviations from polynomially decaying correlation functions are found. Moreover, we provide a generalization of the matrix product state representation for Gaussian states and show that properties hold analogously to the case of finite dimensional spin systems.

\section{INTRODUCTION}

The importance of bosonic Gaussian states arises from two facts. First, they provide a very good description for accessible states of a large variety of physical systems. In fact, every ground and thermal state of a quadratic bosonic Hamiltonian is Gaussian and remains so under quadratic time evolutions. In this way quadratic approximations naturally lead to Gaussian states. Hence, they are ubiquitous in quantum optics as well as in the description of vibrational modes in solid states, ion traps or nanomechanical oscillators.

The second point for the relevance of Gaussian states is that they admit a powerful phase space description which enables us to solve quantum many-body problems which are otherwise (e.g., for spin systems) hardly tractable. In particular, the phase space dimension, and with it the complexity of many tasks, scales linearly rather than exponentially in the number of involved subsystems. For this reason quadratic Hamiltonians and the corresponding Gaussian states also play a paradigmatic role as they may serve as an exactly solvable toy model from which insight into other quantum systems may be gained.

Exploiting the symplectic tools of the phase space description, exact solutions have been found for various problems in quantum information theory as well as in quantum statistical mechanics. In fact, many recent works form a bridge between these two fields as they address entanglement questions for asymptotically large lattices of quadratically coupled harmonic oscillators: the entropic area law [1 $\left[\begin{array}{l}3 \\ 3\end{array}\right]$ has been investigated as well as entanglement statics [4 6], dynamics 7 , 9] and frustration [10, 11].

In the present paper we analytically derive general properties of ground states of translationally invariant quadratic Hamiltonians on a cubic lattice. Moreover, we provide a representation of such states analogous to the matrix product states of finite dimensional spin systems. We start by giving an outlook and a non-technical summary of the main results. The results on the asymptotic scaling of ground state correlations are summarized in Table [1]

We note that related investigations of correlation functions were recently carried out in [12, 13] for finite dimensional spin systems and in [1, 14] for generic harmonic lattices with non-critical finite range interactions. 


\begin{tabular}{|c|c|c|}
\hline interaction & non-critical & critical \\
\hline local & $\begin{array}{c}O^{*}\left(e^{-n / \xi}\right) \\
d=1: \xi \sim \frac{1}{\sqrt{\Delta m^{*}}}=\frac{v}{\Delta}\end{array}$ & \\
\hline$O\left(n^{-\infty}\right)$ & $O\left(n^{-\infty}\right)$ & 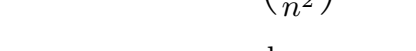 \\
\hline $\begin{array}{c}O\left(\frac{1}{n^{\alpha}}\right) \\
\alpha>2 d+1\end{array}$ & $\begin{array}{l}O\left(\frac{1}{n^{\nu-d}}\right) \\
\alpha>\nu \in \mathbb{N}\end{array}$ & \\
\hline $\begin{array}{c}\frac{c}{n^{\alpha}} \\
d=1\end{array}$ & $\alpha \geq 2: \Theta\left(\frac{1}{n^{\alpha}}\right)$ & $\begin{array}{ll}\alpha=3: & \left\{\begin{array}{l}\Theta\left(\frac{1}{n^{2}}\right), c>0 \\
\Theta\left(\frac{\sqrt{\log n}}{n^{2}}\right), c<0\end{array}\right. \\
\alpha>3: & \Theta\left(\frac{1}{n^{2}}\right)\end{array}$ \\
\hline
\end{tabular}

TABLE I: Summary of the bounds derived in the paper on the asymptotic scaling of ground state correlations, depending on the scaling of the interaction (left column). Here $n$ is the distance between two points (harmonic oscillators) on a cubic lattice of dimension $d$. $O$ denotes upper bounds, $O^{*}$ tight upper bounds, and $\Theta$ the exact asyptotics. The table shows the results for generic interactions - special cases are discussed in the text.

Quadratic Hamiltonians: In Sec. III, we start by introducing some basic results on quadratic Hamiltonians together with the used notation.

Translationally invariant systems: In Sec. III], we show first that every pure translational invariant Gaussian state is point symmetric. This implies that the spectral gap of the symmetrized rather than the original Hamiltonian determines the characteristic properties of the ground state. We provide a general formula for the latter and express its covariance matrix in terms of a product of the inverse of the Fourier transformed spectral function and the Hamiltonian matrix.

Non-critical systems: Sec. IV] shows that if the Hamiltonian is gapped, then the correlations decay according to the interaction: a (super) polynomial decay of the interaction leads to the same (super) polynomial decay for the correlations, and (following Ref. [1]) finite range interactions lead to exponentially decaying correlations.

Correlation length and gap: Sec. $\mathrm{V}$ gives an explicit formula for the correlation length for gapped 1D-Hamiltonians with finite range interactions. The correlation length $\xi$ is expressed in terms of the dominating zero of the complex spectral function, which close to a critical point is in turn determined by the spectral gap $\Delta$ and the effective mass $m^{*}$ at the band gap via $\xi \sim\left(m^{*} \Delta\right)^{-1 / 2}$. When the change in the Hamiltonian is given by a global scaling of the interactions this proves the folk theorem $\xi \sim 1 / \Delta$.

Critical systems: Sec. VI shows that for generic $d$-dimensional critical systems the correlations decay as $1 / n^{d+1}$, where $n$ is the distance between two points on the lattice. Whereas for sufficiently fast decreasing interactions in $d=1$ the asymptotic bound is exactly polynomial, it contains an additional logarithmic correction for $d \geq 2$. Similarly for $d=1$ a logarithmic deviation is found if the interaction decays exactly like $-1 / n^{3}$.

Gaussian matrix product states: Sec. VII provides a representation of Gaussian states in terms of Gaussian matrix product states (GMPS). It is shown that any translational invariant pure state can be approximated by translational invariant GMPS to arbitrary precision, that the correlations of any GMPS decay exponentially, and that every GMPS is the ground state of a local Hamiltonian.

Appendix: Simulating Hamiltonians: We provide a Lemma showing that in $d=1$ every translational invariant Hamiltonian can be simulated by any translational invariant nearest neighbor Hamiltonian supplemented by the set of translational invariant local Hamiltonians. 


\section{QUADRATIC HAMILTONIANS AND THEIR GROUND STATES}

Consider a system of $N$ bosonic modes which are characterized by $N$ pairs of canonical operators $\left(Q_{1}, P_{1}, \ldots, Q_{N}, P_{N}\right)=: R$. The canonical commutation relations (CCR) are governed by the symplectic matrix $\sigma$ via

$$
\left[R_{k}, R_{l}\right]=i \sigma_{k l}, \quad \sigma=\bigoplus_{n=1}^{N}\left(\begin{array}{cc}
0 & 1 \\
-1 & 0
\end{array}\right),
$$

and the system may be equivalently described in terms of bosonic creation and annihilation operators $a_{l}=\left(Q_{l}+i P_{l}\right) / \sqrt{2}$. Quadratic Hamiltonians are of the form

$$
\mathcal{H}=\frac{1}{2} \sum_{k l} H_{k l} R_{k} R_{l}
$$

where the Hamiltonian matrix $H$ is real and positive semidefinite due to the Hermiticity and lower semi-boundedness of the Hamiltonian $\mathcal{H}$. Without loss of generality we neglect linear and constant terms since they can easily be incorporated by a displacement of the canonical operators and a change of the energy offset. Before we discuss the general case we mention some important special instances of quadratic Hamiltonians: a well studied 1D example of this class is the case of nearest neighbor interactions in the position operators of harmonic oscillators on a chain with periodic boundary conditions

$$
\mathcal{H}_{\kappa}=\frac{1}{2} \sum_{i=1}^{N} Q_{i}^{2}+P_{i}^{2}-\kappa Q_{i} Q_{i+1}, \quad \kappa \in[-1,1] .
$$

This kind of spring-like interaction was studied in the context of information transfer [7], entanglement statics [4 6] and entanglement dynamics [9]. Moreover, it can be considered as the discretization of a massive bosonic continuum theory given by the Klein-Gordon Hamiltonian

$$
\mathcal{H}_{\mathrm{KG}}=\frac{1}{2} \int_{-L / 2}^{L / 2}\left[\dot{\phi}(x)^{2}+(\nabla \phi(x))^{2}+m^{2} \phi(x)^{2}\right] d x
$$

where the coupling $\kappa$ is related to the mass $m$ by $\kappa^{-1}=1+\frac{1}{2}\left(\frac{m L}{N}\right)^{2}[5]$. Other finite range quadratic Hamiltonians appear as limiting cases of finite range spin Hamiltonians via the Holstein-Primakoff approximation [15]. In this way the $x y$-spin model with transverse magnetic field can for instance be mapped onto a quadratic bosonic Hamiltonian in the limit of strong polarization where $a \simeq\left(\sigma_{x}+i \sigma_{y}\right) / 2$. Longer range interactions appear naturally for instance in 1D systems of trapped ions. These can either be implemented as Coulomb crystals in Paul traps or in arrays of ion microtraps. When expanding around the equilibrium positions, the interaction between two ions at position $i$ and $j \neq i$ is -in harmonic approximation - of the form $\frac{c Q_{i} Q_{j}}{|i-j|^{3}}$, where $c>0(c<0)$ if $Q_{i}, Q_{j}$ are position operators in radial (axial) direction [16].

Let us now return to the general case and briefly recall the normal mode decomposition [17]: every Hamiltonian matrix can be brought to a diagonal normal form by a congruence transformation with a symplectic matrix $S \in \operatorname{Sp}(2 N, \mathbb{R})=\left\{S \mid S \sigma S^{T}=\sigma\right\}:^{1}$

$$
S H S^{T}=\bigoplus_{i=1}^{I}\left(\begin{array}{cc}
\varepsilon_{i} & 0 \\
0 & \varepsilon_{i}
\end{array}\right) \oplus \bigoplus_{j=1}^{J}\left(\begin{array}{cc}
0 & 0 \\
0 & 1
\end{array}\right), \quad \varepsilon_{i}>0,
$$

where the symplectic eigenvalues $\varepsilon_{i}$ are the square roots of the duplicate nonzero eigenvalues of $\sigma H \sigma^{T} H$. The diagonalizing symplectic transformation $S$ has a unitary representation $U_{S}$

\footnotetext{
${ }^{1}$ Note that we disregard systems where the Hamiltonian contains irrelevant normal modes.
} 
on Hilbert space which transforms the Hamiltonian according to

$$
U_{S} \mathcal{H} U_{S}^{\dagger}=\frac{1}{2} \sum_{i=1}^{I}\left(Q_{i}^{2}+P_{i}^{2}\right) \varepsilon_{i}+\frac{1}{2} \sum_{j=1}^{J} P_{j}^{2}=\sum_{i=1}^{I}\left(a_{i}^{\dagger} a_{i}+\frac{1}{2}\right) \varepsilon_{i}+\frac{1}{2} \sum_{j=1}^{J} P_{j}^{2}
$$

Hence, by Eq. (3) the ground state energy $E_{0}$ and the energy gap $\Delta$ can easily be expressed in terms of the symplectic eigenvalues of the Hamiltonian matrix:

$$
E_{0}=\frac{1}{2} \sum_{i=1}^{I} \varepsilon_{i}, \quad \Delta=\left\{\begin{array}{ll}
\min _{i} \varepsilon_{i}, & J=0 \\
0, & J>0
\end{array} .\right.
$$

The case of a vanishing energy gap $\Delta=0$ is called critical and the respective ground states are often qualitatively different from those of non-critical Hamiltonians. For the Hamiltonian $\mathcal{H}_{\kappa}$, Eq. (1), this happens in the strong coupling limit $|\kappa|=1-\Delta^{2} \rightarrow 1$, and in the case of $1 \mathrm{D}$ Coulomb crystals a vanishing energy gap in the radial modes can be considered as the origin of a structural phase transition where the linear alignment of the ions becomes unstable and changes to a zig-zag configuration [18 20]. Needless to say that these phase transitions appear as well in higher dimensions and for various different configurations [21].

Ground and thermal states of quadratic Hamiltonians are Gaussian states, i.e, states having a Gaussian Wigner distribution in phase space. In the mathematical physics literature they are known as bosonic quasi-free states [22, 23]. These states are completely characterized by their first moments $d_{k}=\operatorname{tr}\left[\rho R_{k}\right]$ (which are w.l.o.g. set to zero in our case) and their covariance matrix (CM)

$$
\gamma_{k l}=\operatorname{tr}\left[\rho\left\{R_{k}-d_{k}, R_{l}-d_{l}\right\}_{+}\right]
$$

where $\{\cdot, \cdot\}_{+}$is the anticommutator. The CM satisfies $\gamma \geq i \sigma$, which expresses Heisenberg's uncertainty relation and is equivalent to the positivity of the corresponding density operator $\rho \geq 0$. In order to find the ground state of a quadratic Hamiltonian, observe that

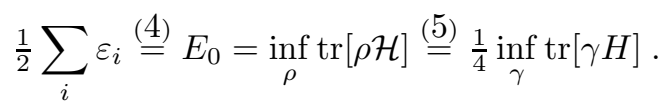

By virtue of Eqs. (23) the infimum is attained for the ground state covariance matrix

$$
\gamma=\lim _{s \rightarrow \infty} S^{T}\left[\bigoplus_{i=1}^{I}\left(\begin{array}{ll}
1 & 0 \\
0 & 1
\end{array}\right) \oplus \bigoplus_{j=1}^{J}\left(\begin{array}{cc}
s & 0 \\
0 & s^{-1}
\end{array}\right)\right] S
$$

which reduces to $\gamma=S^{T} S$ in the non-critical case. Note that the ground state is unique as long as $H$ does not contain irrelevant normal modes [which we have neglected from the very beginning in Eq. (2)].

In many cases it is convenient to change the order of the canonical operators such that $R=\left(Q_{1}, \ldots, Q_{N}, P_{1}, \ldots, P_{N}\right)$. Then the covariance matrix as well as the Hamiltonian matrix can be written in block form

$$
H=\left(\begin{array}{cc}
H_{Q} & H_{Q P} \\
H_{Q P}^{T} & H_{P}
\end{array}\right)
$$

In this representation a quadratic Hamiltonian is particle number preserving iff $H_{Q}=H_{P}$ and $H_{Q P}=-H_{Q P}^{T}$, that is, the Hamiltonian contains only terms of the kind $a_{i}^{\dagger} a_{j}+a_{j}^{\dagger} a_{i}$. In quantum optics terms of the form $a_{i}^{\dagger} a_{j}^{\dagger}$, which are not number preserving, are neglected within the framework of the rotating wave approximation. The resulting Hamiltonians have particular simple ground states:

Theorem 1 (a) The ground state of any particle number preserving Hamiltonian is the vacuum with $\gamma=\mathbb{1}$, and the corresponding ground state energy is given by $E_{0}=\frac{1}{4} \operatorname{tr} H$. 
Proof Number preserving Hamiltonians are most easily expressed in terms of creation and annihilation operators. For this reason we change to the respective complex representation via the transformation

$$
H \mapsto \Omega H \Omega^{T}=\left(\begin{array}{cc}
0 & X \\
\bar{X} & 0
\end{array}\right), \quad \Omega=\frac{1}{\sqrt{2}}\left(\begin{array}{cc}
\mathbb{1} & -i \mathbb{1} \\
\mathbb{1} & i \mathbb{1}
\end{array}\right) .
$$

In this basis $H$ is transformed to normal form via a block diagonal unitary transformation $U \oplus \bar{U}$ which in turn corresponds to an element of the orthogonal subgroup of the symplectic group $\operatorname{Sp}(2 N, \mathbb{R}) \cap \mathrm{SO}(2 N) \simeq \mathrm{U}(N)$ [24]. Hence, the diagonalizing $S$ in Eqs. (217) is orthogonal and since $J=0$ due to particle number conservation, we have $\gamma=S^{T} S=\mathbb{1}$. $E_{0}$ follows then immediately from Eq. (6).

Another important class of quadratic Hamiltonians for which the ground state CM takes on a particular simple form corresponds to the case $H_{Q P}=0$ where there is no coupling between the momentum and position operators:

Theorem 1 (b) For a quadratic Hamiltonian with Hamiltonian matrix $H=H_{Q} \oplus H_{P}$ the ground state energy and the ground state CM are given by

$$
E_{0}=\frac{1}{2} \operatorname{tr}\left[\sqrt{H_{Q}} \sqrt{H_{P}}\right], \gamma=X \oplus X^{-1}, X=H_{Q}^{-1 / 2} \sqrt{H_{Q}^{1 / 2} H_{P} H_{Q}^{1 / 2}} H_{Q}^{-1 / 2} .
$$

Proof Since $\sigma H \sigma^{T} H=H_{P} H_{Q} \oplus H_{Q} H_{P}$, the symplectic eigenvalues of $H$ are given by the eigenvalues of $\sqrt{H_{Q}} \sqrt{H_{P}}$ and thus $E_{0}=\frac{1}{2} \operatorname{tr}\left[\sqrt{H_{Q}} \sqrt{H_{P}}\right]$. Moreover, by the uniqueness of the ground state and the fact that $E_{0}=\frac{1}{4} \operatorname{tr}[\gamma H]$ with $\gamma$ from Eq. (8) we know that $\gamma$ is the ground state CM (as it is an admissible pure state CM by construction).

Finally we give a general formula for the ground state CM in cases where the blocks in the Hamiltonian matrix can be diagonalized simultaneously. This is of particular importance as it applies to all translational invariant Hamiltonians discussed in the following sections.

Theorem 1 (c) Consider a quadratic Hamiltonian for which the blocks $H_{Q}, H_{P}, H_{Q P}$ of the Hamiltonian matrix can be diagonalized simultaneously and in addition $H_{Q P}=H_{Q P}^{T}$. Then with

$$
\begin{aligned}
\hat{\mathcal{E}} & =\sqrt{H_{Q} H_{P}-H_{Q P}^{2}} \quad \text { we have } \\
E_{0} & =\frac{1}{2} \operatorname{tr}[\hat{\mathcal{E}}], \quad \Delta=\lambda_{\min }(\hat{\mathcal{E}}), \quad \gamma=(\hat{\mathcal{E}} \oplus \hat{\mathcal{E}})^{-1} \sigma H \sigma^{T} .
\end{aligned}
$$

Proof Since $\sigma H \sigma^{T} H=\hat{\mathcal{E}}^{2} \oplus \hat{\mathcal{E}}^{2}$ we have indeed $E_{0}=\frac{1}{2} \operatorname{tr}[\hat{\mathcal{E}}]$ and $\Delta=\lambda_{\min }(\hat{\mathcal{E}})$. Positivity $\gamma \geq 0$ is implied by $H \geq 0$ such that we can safely talk about the symplectic eigenvalues of $\gamma$. The latter are, however, all equal to one due to $(\gamma \sigma)^{2}=-\mathbb{1}$ so that $\gamma$ is an admissible pure state CM. Moreover it belongs to the ground state since $\frac{1}{4} \operatorname{tr}[H \gamma]=E_{0}$.

\section{TRANSLATIONALLY INVARIANT SYSTEMS}

Let us now turn towards translationally invariant systems. We consider cubic lattices in $d$ dimensions with periodic boundary conditions. For simplicity we assume that the size of the lattice is $N^{d}$. The system is again characterized by a Hamiltonian matrix $H_{k l}$, where the indices $k, l$, which correspond to two points (harmonic oscillators) on the lattice, are now $d$-component vectors in $\mathbb{Z}_{N}^{d}$. Translational invariance is then reflected by the fact that any matrix element $A_{k l}, A \in\left\{H_{Q}, H_{P}, H_{Q P}\right\}$ depends only on the relative position $k-l$ of the two points on the lattice, and we will therefore often write $A_{k-l}=A_{k l}$. Note that due to the periodic boundary conditions $k-l$ is understood modulo $N$ in each component. Matrices of this type are called circulant, and they are all simultaneously diagonalized via 
the Fourier transform

$$
\begin{aligned}
\mathcal{F}_{\alpha \beta} & =\frac{1}{\sqrt{N}} e^{\frac{2 \pi i}{N} \alpha \beta}, \quad \alpha, \beta \in \mathbb{Z}_{N}, \quad \text { such that } \\
\hat{A} & :=\mathcal{F}^{\otimes d} A \mathcal{F}^{\dagger \otimes d}=\operatorname{diag}\left(\sum_{n \in \mathbb{Z}_{N}^{d}} A_{n} e^{-\frac{2 \pi i}{N} m n}\right)_{m},
\end{aligned}
$$

where $m n$ is the usual scalar product in $\mathbb{Z}_{N}^{d}$. It follows immediately that all circulant matrices mutually commute.

In the following, we will show that we can without loss of generality restrict ourselves to point-symmetric Hamiltonians, i.e., those for which $H_{Q P}=H_{Q P}^{T}$ (which means that $\mathcal{H}$ contains only pairs $\left.Q_{k} P_{l}+Q_{l} P_{k}\right)$. For dimension $d=1$ this is often called reflection symmetry.

Theorem 2 Any translationally invariant pure state $C M \Gamma$ is point symmetric.

Proof For the proof, we use that any pure state covariance matrix can be written as

$$
\Gamma=\left(\begin{array}{cc}
\Gamma_{Q} & \Gamma_{Q P} \\
\Gamma_{Q P}^{T} & \Gamma_{P}
\end{array}\right)=\left(\begin{array}{cc}
X & X Y \\
Y X & X^{-1}+Y X Y
\end{array}\right),
$$

where $X \geq 0$ and $Y$ is real and symmetric [25]. From translational invariance, it follows that all blocks and thus $X$ and $Y$ have to be circulant and therefore commute. Hence, $\Gamma_{Q P}=X Y=Y X=\Gamma_{Q P}^{T}$, i.e., $\Gamma$ is point symmetric.

Let $\mathcal{P}: \mathbb{Z}_{N}^{d} \rightarrow \mathbb{Z}_{N}^{d}$ be the reflection on the lattice and define the symmetrization operation $\mathcal{S}(A)=\frac{1}{2}(A+\mathcal{P} A \mathcal{P})$ such that by the above theorem $\mathcal{S}(\gamma)=\gamma$ for every translational invariant pure state CM. Then due to the cyclicity of the trace we have for any translational invariant Hamiltonian

$$
\inf _{\gamma} \operatorname{tr}[H \gamma]=\inf _{\gamma} \operatorname{tr}[\mathcal{S}(H) \gamma] .
$$

Hence, the point-symmetrized Hamiltonian $\mathcal{S}(H)$, which differs from $H$ by the off-diagonal block $\mathcal{S}\left(H_{Q P}\right)=\frac{1}{2}\left(H_{Q P}+H_{Q P}^{T}\right)$ has both the same ground state energy and the same ground state as $H$. Together with Theorem 1c this leads us to the following:

Theorem 3 Consider any translationally invariant quadratic Hamiltonian. With $\hat{\mathcal{E}}=$ $\left[H_{Q} H_{P}-\frac{1}{4}\left(H_{Q P}+H_{Q P}^{T}\right)^{2}\right]^{1 / 2}$ the ground state $C M$ and the corresponding ground state energy are given by

$$
E_{0}=\frac{1}{2} \operatorname{tr}[\hat{\mathcal{E}}], \quad \gamma=(\hat{\mathcal{E}} \oplus \hat{\mathcal{E}})^{-1} \sigma \mathcal{S}(H) \sigma^{T} .
$$

It is important to note that the energy gaps of $H$ and $\mathcal{S}(H)$ will in general be different. In particular $H$ might be gapless while $\mathcal{S}(H)$ is gapped. However, as we will see in the following sections, the properties of $\gamma$ depend on the gap $\Delta=\lambda_{\min }(\hat{\mathcal{E}})$ of the symmetrized Hamiltonian rather than on that of the original $H$. For this reason we will in the following for simplicity assume $H_{Q P}=H_{Q P}^{T}$. By Thm. 3 all results can then also be applied to the general case without point symmetry if one only keeps in mind that $\Delta$ is the gap corresponding to $\mathcal{S}(H)$.

Note that the eigenvalues of $\hat{\mathcal{E}}$ are the symplectic eigenvalues of $\mathcal{S}(H)$, i.e., $\mathcal{E}=\mathcal{F}^{\otimes d} \hat{\mathcal{E}} \mathcal{F}^{\dagger} \otimes d$ is the excitation spectrum of the Hamiltonian. This is the reason for the notation where $\mathcal{E}$ resides in Fourier space and $\hat{\mathcal{E}}$ in real space, which is differs from the normal usage of the hat throughout the paper.

Correlation functions. According to Eqs. (910[11) we have to compute the entries of functions of matrices in order to learn about the entries of the covariance matrix. This is most conveniently done by a double Fourier transformation, where one uses that $\hat{f(M)}=f(\hat{M})$, and we find 


$$
[f(M)]_{n m}=\frac{1}{N^{d}} \sum_{r, s} e^{-\frac{2 \pi i}{N} n r}[f(\hat{M})]_{r s} e^{\frac{2 \pi i}{N} s m} .
$$

As we consider translationally invariant systems, $M$ is circulant and thus $\hat{M}$ is diagonal. We define the function

$$
\hat{M}(\phi)=\sum_{n \in \mathbb{Z}_{N}^{d}} M_{n} e^{-i n \phi}
$$

such that $\hat{M}(2 \pi r / N)=\hat{M}_{r, r}$. As $f(M)$ is solely determined by its first row, we can write

$$
[f(M)]_{n}=\frac{1}{N^{d}} \sum_{r \in \mathbb{Z}_{N}^{d}} e^{2 \pi i n r / N} f(\hat{M}(2 \pi r / N)) .
$$

In the following we will use the index $n \in \mathbb{Z}^{d}$ for the relative position of two points on the lattice. Their distance will be measured either by the $l_{1}, l_{2}$ or $l_{\infty}$ norm. Since we are considering finite dimensional lattices these are all equivalent for our purpose and we will simply write $\|n\|$. In the thermodynamic limit $N \rightarrow \infty$, the sum in Eq. (14) converges to the integral

$$
[f(M)]_{n}=\frac{1}{(2 \pi)^{d}} \int_{\mathcal{T}^{d}} \mathrm{~d} \phi f(\hat{M}(\phi)) e^{i n \phi} \quad \text { with } \hat{M}(\phi)=\sum_{n \in \mathbb{Z}^{d}} M_{n} e^{-i n \phi},
$$

where $\mathcal{T}^{d}$ is the $d$-dimensional torus, i.e., $[0,2 \pi]^{d}$ with periodic boundary conditions. The convergence holds as soon as $\sum\left|M_{n}\right|<\infty$ [which holds e.g. for $M_{n}=O\left(\|n\|^{-\alpha}\right)$ with some $\alpha>d]$ and $f$ is continuous on an open interval which contains the range of $\hat{M}$.

From the definition (15) of $\hat{M}$, it follows that $\hat{M} \in \mathscr{C}^{k}\left(\mathcal{T}^{d}\right)$ (the $n$ times continuously differentiable functions on $\mathcal{T}^{d}$ ) whenever the entries $M_{n}$ decay at least as fast as $\|n\|^{-\alpha}$ for some $\alpha>k+d$, since then the sum of the derivatives converges uniformly. Particularly, if the entries of $M$ decay faster than any polynomial, then $\hat{M} \in \mathscr{C}^{\infty}\left(\mathcal{T}^{d}\right)$. In the following the most important function of the type $f \circ \hat{M}$ will be the spectral function

$$
\mathcal{E}(\phi)=\sqrt{\sum_{n \in \mathbb{Z}^{d}} e^{-i n \phi}\left(\left[H_{Q} H_{P}\right]_{n}-\left[H_{Q P}^{2}\right]_{n}\right)} .
$$

Asymptotic notation. As the main issue of this paper is the asymptotic scaling of correlations, we use the Landau symbols $o, O$, and $\Theta$, as well as the symbol $O^{*}$ for tight bounds:

- $f(x)=o(g(x))$ means $\lim _{x \rightarrow \infty} \frac{f(x)}{g(x)}=0$, i.e., $f$ vanishes strictly faster than $g$ for $x \rightarrow \infty$;

- $f(x)=O(g(x))$, if $\limsup _{x \rightarrow \infty}\left|\frac{f(x)}{g(x)}\right|$ is finite, i.e., $f$ vanishes at least as fast as $g$;

- $f(x)=\Theta(g(x))$, if $f(x)=O(g(x))$ and $g(x)=O(f(x))$ (i.e., exact asymptotics);

- $f(x)=O^{*}(g(x))$, if $f(x)=O(g(x))$ but $f(x) \neq o(g(x))$, i.e., $g$ is a tight bound on $f .^{2}$ If $f$ is taken from a set (e.g., those function consistent with the assumptions of a theorem) we will write $f=O^{*}(g)$ if $g$ is a tight bound for at least one $f$ (i.e., the best possible universal bound under the given assumptions).

If talking about Hamiltonians, the scaling is meant to hold for all blocks, e.g., if the interaction vanishes as $O\left(\|n\|^{-\alpha}\right)$ for $n \rightarrow \infty$, this holds for all the blocks $H_{Q}, H_{P}$, and $H_{Q P}=H_{P Q}^{T}$. The same holds for covariance matrices in the non-critical case. By the shorthand notation $f(n)=o\left(\|n\|^{-\infty}\right)$, we mean that $f(n)=o\left(\|n\|^{-\alpha}\right) \forall \alpha>0$. Note finally that the Landau symbols are also used in (Taylor) expansions around a point $x_{0}$ where the considered limit is $x \rightarrow x_{0}$ rather than $x \rightarrow \infty$.

2 In order to see the difference to $\Theta$, take an $f(x)=g(x)$ for even $x, f(x)=0$ for odd $x, x \in \mathbb{N}$. Although $f$ does not bound $g$, thus $f(x) \neq O(g(x))$, the bound $g$ is certainly tight. A situation like this is met, e.g., in Theorem 9 where the correlations oscillate within an exponentially decaying envelope. 


\section{NON-CRITICAL SYSTEMS}

In this section, we analyze the ground state correlations of non-critical systems, i.e., those which exhibit an energy gap $\Delta>0$ between the ground an the first excited state. Simply speaking, we will show that the decay of correlations reflects the decay of the interaction. While local (super-polynomially decaying) interactions imply exponentially (superpolynomially) decaying correlations, a polynomial decay of interactions will lead to the same polynomial law for the correlations.

According to Theorem 3, we will consider a translationally invariant system with a pointsymmetric Hamiltonian $\left(H_{Q P}=H_{Q P}^{T}\right)$. Following (1011), we have to determine the entries of $\left(\hat{\mathcal{E}}^{-1} \oplus \hat{\mathcal{E}}^{-1}\right) \sigma H \sigma^{T}$, with $\hat{\mathcal{E}}=\left(H_{Q} H_{P}+H_{Q P}^{2}\right)^{1 / 2}$. In Lemma 4 we will first show that it is possible to consider the two contributions independently, and as the asymptotics of $\sigma H \sigma^{T}$ is known, we only have to care about the entries of $\hat{\mathcal{E}}^{-1}$, i.e., we have to determine the asymptotic behavior of the integral

$$
\left(\hat{\mathcal{E}}^{-1}\right)_{n}=\frac{1}{(2 \pi)^{d}} \int_{\mathcal{T}^{d}} \mathrm{~d} \phi \mathcal{E}^{-1}(\phi) e^{i n \phi} \quad \text { where } \quad \mathcal{E}=\left(\hat{H}_{Q} \hat{H}_{P}+\hat{H}_{Q P}^{2}\right)^{1 / 2}
$$

Lemma 4 Given two asymptotic circulant matrices $A, B$ in d dimensions with polynomially decaying entries, $A_{n}=O\left(\|n\|^{-\alpha}\right), B_{n}=O\left(\|n\|^{-\beta}\right), \alpha, \beta>d$. Then

$$
(A B)_{n}=O^{*}\left(\|n\|^{-\mu}\right), \quad \mu:=\min \{\alpha, \beta\} .
$$

Proof With $Q_{\eta}(n):=\min \left\{1,\|n\|^{-\eta}\right\}$, we know that $\left|A_{n}\right|=O\left(Q_{\alpha}\right)$ and $\left|B_{n}\right|=O\left(Q_{\beta}\right)$, and

$$
\left|(A B)_{n}\right|=\left|\sum_{j} A_{0, j} B_{j, n}\right| \leq \sum_{j}\left|A_{j}\right|\left|B_{n-j}\right|=O\left(\sum_{j} Q_{\alpha}(j) Q_{\beta}(n-j)\right) .
$$

We consider only one half space $\|j\| \leq\|n-j\|$, where we bound $Q_{\beta}(n-j) \leq Q_{\beta}(n / 2)$. As $Q_{\alpha}(j)$ is summable, the contribution of this half-plane is $O\left(Q_{\beta}(n / 2)\right)$. The other half-plane gives the same result with $\alpha$ and $\beta$ interchanged, which proves the bound, while tightness follows by taking all $A_{n}, B_{n}$ positive.

We now determine the asymptotics of $\left(\hat{\mathcal{E}}^{-1}\right)_{n}$ for different types of Hamiltonians.

Lemma 5 For non-critical systems with rapidly decaying interactions, i.e., as o $\left(\|n\|^{-\infty}\right)$, the entries of $\hat{\mathcal{E}}^{-1}$ decay rapidly as well. That is,

$$
\Delta>0 \Rightarrow\left(\hat{\mathcal{E}}^{-1}\right)_{n}=o\left(\|n\|^{-\infty}\right)
$$

Proof As the interactions decay as $o\left(\|n\|^{-\infty}\right), \hat{H}_{\bullet} \in \mathscr{C}^{\infty}\left(\mathcal{T}^{d}\right)(\bullet=Q, P, P Q)$, and thus $\mathcal{E}^{2}=\hat{H}_{Q} \hat{H}_{P}+\hat{H}_{Q P}^{2} \in \mathscr{C}^{\infty}\left(\mathcal{T}^{d}\right)$. Since the system is gapped, i.e., $\mathcal{E} \geq \Delta>0$, it follows that also $g:=\mathcal{E}^{-1} \in \mathscr{C}^{\infty}\left(\mathcal{T}^{d}\right)$. For the proof, we need to bound

$$
\left(\hat{\mathcal{E}}^{-1}\right)_{n}=\frac{1}{(2 \pi)^{d}} \int_{\mathcal{T}^{d}} \mathrm{~d} \phi g(\phi) e^{i n \phi}
$$

by $\|n\|^{-\kappa}$ for all $\kappa \in \mathbb{N}$. First, let us have a look at the one-dimensional case. By integration by parts, we get

$$
\left(\hat{\mathcal{E}}^{-1}\right)_{n}=\frac{1}{2 \pi}\left[\frac{1}{i n} g(\phi) e^{i n \phi}\right]_{\phi=-\pi}^{\pi}-\frac{1}{2 \pi i n} \int_{-\pi}^{\pi} \mathrm{d} \phi g^{\prime}(\phi) e^{i n \phi},
$$

where the first part vanishes due to the periodicity of $g$. As $g \in \mathscr{C}^{\infty}\left(\mathcal{T}^{1}\right)$, the integration by parts can be iterated arbitrarily often and all the brackets vanish, such that after $\kappa$ iterations,

$$
\left(\hat{\mathcal{E}}^{-1}\right)_{n}=\frac{1}{2 \pi(i n)^{\kappa}} \int_{-\pi}^{\pi} \mathrm{d} \phi g^{(\kappa)}(\phi) e^{i n \phi}
$$


As $g^{(\kappa)}(\phi)$ is continuous, the integral can be bounded by $\int\left|g^{(\kappa)}(\phi)\right| \mathrm{d} \phi=: C_{\kappa}<\infty$, such that finally

$$
\left|\left(\hat{\mathcal{E}}^{-1}\right)_{n}\right| \leq \frac{C_{\kappa}}{n^{\kappa}} \quad \forall \kappa \in \mathbb{N},
$$

which completes the proof of the one-dimensional case.

The extension to higher dimensions is straightforward. For a given $n=\left(n_{1}, \ldots, n_{d}\right)$, integrate by parts with respect to the $\phi_{i}$ for which $\left|n_{i}\right|=\|n\|_{\infty}$; we assume $i=1$ without loss of generality. As $g\left(\cdot, \phi_{2}, \ldots, \phi_{d}\right) \in \mathscr{C}^{\infty}\left(\mathcal{S}^{1}\right)$, the same arguments as in the $1 \mathrm{D}$ case show

$$
\left|\left(\hat{\mathcal{E}}^{-1}\right)_{n}\right| \leq \frac{1}{(2 \pi)^{d}\left|n_{1}\right|^{\kappa}} \int_{\mathcal{T}^{d}}\left|\frac{\partial^{\kappa}}{\partial \phi_{1}^{\kappa}} g(\phi)\right| \mathrm{d} \phi=\frac{C_{\kappa}}{\|n\|_{\infty}^{\kappa}} .
$$

For systems with local interactions, a stronger version of Lemma 5 can be obtained:

Lemma 6 For a system with finite range interaction, the entries of $\hat{\mathcal{E}}^{-1}$ decay exponentially.

This has been proven in [1] for Hamiltonians of the type $H=V \oplus \mathbb{1}$, exploiting a result on functions of banded matrices [26]. Following Eqs. (9]11) the generalization to arbitrary translational invariant Hamiltonians is straightforward by replacing $V$ with $H_{Q} H_{P}-H_{Q P}^{2}$. In fact, it has been shown recently that the result even extends to non translational invariant Hamiltonians of the form in Theorem $1 \mathrm{~b}$ [14].

Finally, we consider systems with polynomially decaying interaction.

Lemma 7 For a $1 D$ lattice with $H=V \oplus \mathbb{1}>0$ and an exactly polynomially decaying interaction

$$
V_{i j}=\left\{\begin{array}{lll}
i=j & : & a \\
i \neq j & : & \frac{b}{|i-j|^{\nu}}
\end{array} \quad, 2 \leq \nu \in \mathbb{N},\right.
$$

$\hat{\mathcal{E}}^{-1}$ decays polynomially with the same exponent, $\left(\hat{\mathcal{E}}^{-1}\right)_{n}=\left(V^{1 / 2}\right)_{n}=\Theta\left(|n|^{-\nu}\right)$.

Hamiltonians of this type appear, e.g., for the vibrational degrees of freedom of ions in a linear trap, where $\nu=3$.

Proof We need to estimate $\left(\hat{\mathcal{E}}^{-1}\right)_{n} \stackrel{\underline{\underline{9}}}{=}\left(V^{-1 / 2}\right)_{n}=\frac{1}{2 \pi} \int_{0}^{2 \pi} \hat{V}^{-1 / 2}(\phi) e^{i n \phi} \mathrm{d} \phi$. Note that

$$
\hat{V}(\phi)=a+2 b \sum_{n=1}^{\infty} \frac{\cos (n \phi)}{n^{\nu}}=a+2 b \operatorname{Re}\left[\operatorname{Li}_{\nu}\left(e^{i \phi}\right)\right]>0
$$

where $\operatorname{Li}_{\nu}(z)=\sum_{n \geq 1} z^{n} / n^{\nu}$ is the polylogarithm. The polynomial decay of coefficients implies $\hat{V} \in \mathscr{C}^{\nu-2}\left(\mathcal{S}^{1}\right)$, and as the system is non-critical, $\hat{V}^{-1 / 2} \in \mathscr{C}^{\nu-2}\left(\mathcal{S}^{1}\right)$. As $\operatorname{Li}_{\nu}$ has an analytic continuation to $\mathbb{C} \backslash[1 ; \infty), \hat{V} \in \mathscr{C}^{\infty}((0 ; 2 \pi))$ and thus $\hat{V}^{-1 / 2} \in \mathscr{C}^{\infty}((0 ; 2 \pi))$. We can therefore integrate by parts $\nu-1$ times, and as all brackets vanish due to periodicity, we obtain

$$
\left(\hat{\mathcal{E}}^{-1}\right)_{n}=\frac{1}{2 \pi(i n)^{\nu-1}} \int_{0}^{2 \pi}\left[\frac{\mathrm{d}^{\nu-1}}{\mathrm{~d} \phi^{\nu-1}} \hat{V}^{-1 / 2}(\phi)\right] e^{i n \phi} \mathrm{d} \phi
$$

and

$$
\frac{\mathrm{d}^{\nu-1}}{\mathrm{~d} \phi^{\nu-1}} \hat{V}^{-1 / 2}(\phi)=-\frac{\hat{V}^{(\nu-1)}(\phi)}{2 \hat{V}(\phi)^{3 / 2}}+\frac{3(\nu-2) \hat{V}^{(\nu-2)}(\phi) \hat{V}^{(1)}(\phi)}{4 \hat{V}(\phi)^{5 / 2}}+g(\phi) .
$$

Note that the second term only appears if $\nu \geq 3$, and $g$ only if $\nu \geq 4$. As $g(\phi) \in \mathscr{C}^{1}\left(\mathcal{S}^{1}\right)$, its Fourier coefficients vanish as $O\left(n^{-1}\right)$, as can be shown by integrating by parts. The 
second term can be integrated by parts as well, the bracket vanishes due to continuity, and we remain with

$$
\frac{1}{i n} \int_{0}^{2 \pi}\left[\frac{3(\nu-2) \hat{V}^{(\nu-1)}(\phi) \hat{V}^{(1)}(\phi)}{4 \hat{V}(\phi)^{5 / 2}}+h(\phi)\right] e^{i n \phi} \mathrm{d} \phi
$$

with $h \in \mathscr{C}\left(\mathcal{S}^{1}\right)$. [For $\nu=3$, a factor 2 appears as $\left(\hat{V}^{(1)}\right)^{\prime}=\hat{V}^{(\nu-1)}$.] As we will show later, $\hat{V}^{(\nu-1)}$ is absolutely integrable, hence the integral exists, and thus the Fourier coefficients of the second term in Eq. (20) vanish as $O\left(n^{-1}\right)$ as well. Finally, it remains to bound

$$
\int_{0}^{2 \pi} \frac{\hat{V}^{(\nu-1)}(\phi)}{2 \hat{V}(\phi)^{3 / 2}} e^{i n \phi} \mathrm{d} \phi
$$

As $\operatorname{Li}_{\nu}^{\prime}(x)=\operatorname{Li}_{\nu-1}(x) / x$, it follows from Eq. (18) that

$$
V^{(\nu-1)}(\phi)=2 b \operatorname{Re}\left[i^{\nu-1} \operatorname{Li}_{1}\left(e^{i \phi}\right)\right]=2 b \operatorname{Re}\left[-i^{\nu-1} \log \left(1-e^{i \phi}\right)\right],
$$

where the last step is from the definition of $\mathrm{Li}_{1}$.

We now distinguish two cases. First, assume that $\nu$ is even. Then,

$$
V^{(\nu-1)}(\phi) \propto \operatorname{Im} \log \left(1-e^{i \phi}\right)=\arg \left(1-e^{i \phi}\right)=(\phi-\pi) / 2
$$

on $(0 ; 2 \pi)$, hence the integrand in Eq. (21) is bounded and has a bounded derivative, and by integration by parts, the integral Eq. (21) is $O\left(n^{-1}\right)$. In case $\nu$ is odd we have

$$
V^{(\nu-1)}(\phi) \propto \operatorname{Re} \log \left(1-e^{i \phi}\right)=\log \left|1-e^{i \phi}\right|=\log (2 \sin (\phi / 2))
$$

on $(0 ; 2 \pi)$. With $h(\phi):=\hat{V}^{-3 / 2}(\phi) / 2$, the integrand in Eq. (21) can be written as

$$
\hat{V}^{(\nu-1)}(\phi) h(\phi) \propto \log (2 \sin (\phi / 2)) h(0)+\log (2 \sin (\phi / 2))[h(\phi)-h(0)] .
$$

The first term gives a contribution proportional to

$$
\int_{0}^{2 \pi} \log (2 \sin (\phi / 2)) \cos (n \phi) \mathrm{d} \phi=-\frac{1}{2 n}
$$

as it is the back-transform of $-\frac{1}{2} \sum_{n \geq 1} \cos (n \phi) / n$. For the second term, note that $h \in$ $\mathscr{C}^{1}\left(\mathcal{S}^{1}\right)$ for $\nu \geq 3$ and thus $h(\phi)-h(0)=h^{\prime}(0) \phi+o(\phi)$ by Taylor's theorem. Therefore, the log singularity vanishes, and we can once more integrate by parts. The derivative is

$$
\frac{1}{2} \cot (\phi / 2)[h(\phi)-h(0)]+\log (2 \sin (\phi / 2)) h^{\prime}(\phi) \text {. }
$$

In the left part, the $1 / \phi$ singularity of $\cot (\phi / 2)$ is cancelled out by $h(\phi)-h(0)=O(\phi)$, and the second part is integrable as $h^{\prime} \in \mathscr{C}\left(\mathcal{S}^{1}\right)$, so that the contribution of the integral (21) is $O\left(n^{-1}\right)$ as well.

In order to show that $n^{-\nu}$ is also a lower bound on $\left(\hat{V}^{-1 / 2}\right)_{n}$, one has to analyze the asymptotics more carefully. Using the Riemann-Lebesgue lemma - which says that the Fourier coefficients of absolutely integrable functions are $o(1)$ - one finds that all terms in (19) vanish as $o\left(1 / n^{\nu}\right)$, except for the integral (21). Now for even $\nu$, (21) can be integrated by parts, and while the brackets give a $\Theta\left(n^{-\nu}\right)$ term, the remaining integral is $o\left(n^{-\nu}\right)$, which proves that $\left(\hat{V}^{-1 / 2}\right)_{n}=\Theta\left(n^{-\nu}\right)$. For odd $\nu$, on the other hand, the first part of (22) gives exactly a polynomial decay, while the contributions from the second part vanishes as $o\left(n^{-\nu}\right)$, which proves $\left(\hat{V}^{-1 / 2}\right)_{n}=\Theta\left(n^{-\nu}\right)$ for odd $\nu$ as well.

Generalizations of Lemma 7. The preceding lemma can be extended to non-integer exponents $\alpha \notin \mathbb{N}$ : if $V_{n} \propto n^{-\alpha}, n \neq 0$, then $\left(\hat{\mathcal{E}}^{-1}\right)_{n}=O\left(n^{-\alpha}\right)$.

For the proof, define $\alpha=\nu+\varepsilon, \nu \in \mathbb{N}, 0<\varepsilon<1$. Then $\hat{V} \in \mathscr{C}^{\nu-1}\left(\mathcal{S}^{1}\right), \hat{V} \in \mathscr{C}^{\infty}((0 ; 2 \pi))$, and one can integrate by parts $\nu$ times, where all brackets vanish. What remains is to 
bound the Fourier integral of the $\nu^{\prime}$ th derivative of $\hat{V}^{-1 / 2}$ by $n^{-\varepsilon}$. An upper bound can be established by noting that $\left|\hat{V}^{(\nu)}(\phi)\right| \leq\left|\operatorname{Li}_{\varepsilon}\left(e^{i \phi}\right)\right|=O\left(\phi^{\varepsilon-1}\right)$ and $\left|\hat{V}^{(\nu+1)}(\phi)\right|=O\left(\phi^{\varepsilon-2}\right)$. It follows that all contributions in the Fourier integral except the singularity from $\hat{V}^{(\nu)}$ lead to $o(1 / n)$ contributions as can be shown by another integration by parts. In order to bound the Fourier integral of the $O\left(\phi^{\varepsilon-1}\right)$ term, split the Fourier integral at $\frac{1}{n}$. The integral over $\left[0 ; \frac{1}{n}\right]$ can be directly bounded by $n^{-\varepsilon}$, while for $\left[\frac{1}{n} ; 1\right]$, an equivalent bound can be established after integration by parts, using $\hat{V}^{(\nu+1)}=O\left(\phi^{\varepsilon-2}\right)$. This method is discussed in more detail in the proof of Theorem 15, following Eq. (44).

The proof that $n^{-\varepsilon}$ is also a lower bound to $\left(\hat{\mathcal{E}}^{-1}\right)_{n}$ is more involved. From a series expansion of $\hat{V}$ and its derivatives, it can be seen that it suffices to bound the sine and cosine Fourier coefficients of $\phi^{\varepsilon-1}$ from below. As in the proof of Theorem 14 this is accomplished by splitting the integral into single oscillations of the sine or cosine and bounding each part by the derivative of $\phi^{\varepsilon-1}$.

For polynomially bounded interactions $V_{n}=O\left(n^{-\alpha}\right), \alpha>1$, not very much can be said without further knowledge. With $\nu<\alpha, \nu \in \mathbb{N}$ the largest integer strictly smaller than $\alpha$, we know that $\hat{V} \in \mathscr{C}^{\nu-1}\left(\mathcal{S}^{1}\right)$. Thus, one can integrate by parts $\nu-1$ times, the brackets vanish, and the remaining Fourier integral is $o(1)$ using the Riemann-Lebesgue lemma. It follows that $\left(\hat{\mathcal{E}}^{-1}\right)_{n}=o\left(n^{-(\nu-1)}\right)$. In contrast to the case of an exactly polynomial decay, this can be extended to higher spatial dimensions $d>1$ by replacing $\nu-1$ with $\nu-d$, which yields $\left(\hat{\mathcal{E}}^{-1}\right)_{n}=o\left(n^{-(\nu-d)}\right)$.

We now use the preceding lemmas about the entries of $\hat{\mathcal{E}}^{-1}$ (Lemma 57) to derive corresponding results on the correlations of ground states of non-critical systems.

Theorem 8 For systems with $\Delta>0$, the following holds:

(i) If the Hamiltonian $H$ has finite range, the ground state correlations decay exponentially.

(ii) If $H$ decays as $o\left(\|n\|^{-\infty}\right)$, the ground state correlations decay as $o\left(\|n\|^{-\infty}\right)$ as well.

(iii) For a $1 D$ system with $H=V \oplus \mathbb{1}$ where $V$ decays with a power law $|n|^{-\nu}, \nu \geq 2$, the ground state correlations decay as $\Theta\left(|n|^{-\nu}\right)$.

Proof In all cases, we have to find the scaling of the ground state $\gamma$ which is the product $\gamma=\left(\hat{\mathcal{E}}^{-1} \oplus \hat{\mathcal{E}}^{-1}\right) \sigma H \sigma^{T}$, Eq. (10). Part (i) follows directly from Lemma 6, as multiplying with a finite-range $\sigma H \sigma^{T}$ doesn't change the exponential decay, while (ii) follows from Lemma 5 , the $o\left(\|n\|^{-\infty}\right)$ decay of $\sigma H \sigma^{T}$, and Lemma 4. To show (iii), note that for $H=V \oplus \mathbb{1}$, the ground state is $\gamma=V^{-1 / 2} \oplus V^{1 / 2}$, and from Lemma 7 O $O\left(n^{-\nu}\right)$ follows. For $\hat{V}^{-1 / 2}$, Lemma 7 also includes that the bound is exact, while for $\hat{V}^{1 / 2}$, it can be shown by transferring the proof of the lemma one-to-one.

Note that a simple converse of Theorem 8 always holds: for each translationally invariant pure state $\mathrm{CM} \gamma$, there exists a Hamiltonian $H$ with the same asymptotic behavior as $\gamma$ such that $\gamma$ is the ground state of $H$. This can be trivially seen by choosing $H=\sigma \gamma \sigma^{T}$.

\section{CORRELATION LENGTH AND GAP}

In this section, we consider one-dimensional chains with local gapped Hamiltonians. We compute the correlation length for these systems and use this result to derive a relation between correlation length and gap.

Theorem 9 Consider a non-critical 1D chain with a local Hamiltonian. Define the complex extension of the spectral function $\mathcal{E}(\phi)=\left[\sum_{n=0}^{L} c_{n} \cos (n \phi)\right]^{1 / 2}$ in Eq. (16) as

$$
g(z):=\sum_{n=0}^{L} c_{n} \frac{z^{n}+z^{-n}}{2}
$$




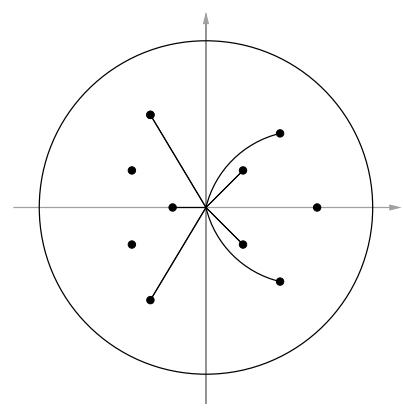

FIG. 1: Sample arrangement of branch cuts and poles of $\sqrt{g}$ inside the unit circle. From each odd order zero of $g$, a branch cut emerges. All cuts go to 0 where they cancel with another cut. In case their number is odd, there is an additional branch point at 0 cancelling the last cut. In case two zeros are on a line to the origin, the cuts are chosen curved. The integral of $\sqrt{g}$ around the unit circle is equal to the integral around the cuts, plus integrals around the residues which originate from the even order zeros of $g$.

such that $g\left(e^{i \phi}\right)=\mathcal{E}^{2}(\phi) \stackrel{\sqrt[90]{=}}{=} \hat{H}_{Q}(\phi) \hat{H}_{P}(\phi)-\hat{H}_{Q P}^{2}(\phi)$ and let $\tilde{z}$ be zero of $g$ with the largest magnitude smaller than one. Then, the correlation length

$$
\xi=-\frac{1}{\log |\tilde{z}|}
$$

determines the asymptotic scaling of the correlations which is given by

- $O^{*}\left(e^{-n / \xi} / \sqrt{n}\right)$, if $\tilde{z}$ is a zero of order one,

- $O^{*}\left(e^{-n / \xi}\right)$, if $\tilde{z}$ is a zero of even order,

- $o\left(e^{-n /(\xi+\varepsilon)}\right)$ for all $\varepsilon>0$, if $\tilde{z}$ is a zero of odd order larger than one.

For the nearest neighbor interaction Hamiltonian $\mathcal{H}_{\kappa}$ from Eq. (1) one has for instance $\mathcal{E}(\phi)=\sqrt{1-\kappa \cos (\phi)}$, so that $g$ has simple zeros at $z_{0}=\left(1 \pm \sqrt{1-\kappa^{2}}\right) / \kappa$. Therefore $\tilde{z}=\left(1-\sqrt{1-\kappa^{2}}\right) / \kappa$, and the correlations decay as $\Theta\left(e^{-n / \xi} / \sqrt{n}\right)$ where $\xi=-1 / \log |\tilde{z}|$.

Proof For local Hamiltonians, the correlations decay as the matrix elements of $\hat{\mathcal{E}}^{-1}$ [Eq. (10)]. By Fourier transforming (9), $\mathcal{E}(\phi)=\sqrt{g\left(e^{i \phi)}\right.}$, with $g\left(e^{i \phi}\right)=\hat{H}_{Q}(\phi) \hat{H}_{P}(\phi)-$ $\hat{H}_{Q P}^{2}(\phi)=\sum_{n=0}^{L} c_{n} \cos (n \phi)$ an even trigonometric polynomial (we assume $c_{L} \neq 0$ without loss of generality), and $\min \left(g\left(e^{i \phi}\right)\right)=\Delta^{2}$. We have to compute

$$
\left(\hat{\mathcal{E}}^{-1}\right)_{n}=\frac{1}{2 \pi} \int_{0}^{2 \pi} \frac{1}{\mathcal{E}(\phi)} e^{i n \phi} \mathrm{d} \phi=\frac{1}{2 \pi i} \int_{\mathcal{S}^{1}} \frac{z^{n-1}}{\sqrt{g(z)}} \mathrm{d} z
$$

where $\mathcal{S}^{1}$ is the unit circle. The function $g(z)$ has a pole of order $L$ at zero and $2 L$ zeros altogether. Since $\min (g(\phi))=\Delta^{2}>0, g$ has no zeros on the unit circle. As $g(z)=g(1 / z)$, the zeros come in pairs, and $L$ of them are inside the unit circle. Also, the conjugate of a zero is a zero as well. From each zero with odd multiplicity emerges a branch cut of $\sqrt{g(z)}$. We arrange all the branch cuts inside the unit circle such that they go straight to the middle where they annihilate with another cut. In case $L$ is odd, the last cut is annihilated by the singularity of $\sqrt{g(z)}$ at 0 . If two zeros lie on a line, one cut curves slightly. A sample arrangement is shown in Fig. 1 .

Following Cauchy's theorem, the integral can be decomposed into integrals along the different branch cuts and around the residues of $1 / \sqrt{g}$, and one has to estimate the contributions from the different types of zeros of $g$. The simplest case is given by zeros $z_{0}$ with even multiplicity $2 m$. In that case, define $h(z):=g(z) /\left(z-z_{0}\right)^{2 m}$ which has no zero around $z_{0}$. The contribution from $z_{0}$ to the correlations is then given by the residue at $z_{0}$ and is

$$
\left.\frac{1}{(m-1) !} \frac{\mathrm{d}^{m-1}}{\mathrm{~d} z^{m-1}}\left(\frac{z^{n-1}}{\sqrt{h(z)}}\right)\right|_{z=z_{0}} \propto z_{0}^{n-(m-1)}
$$


for $n-(m-1)>0$, i.e., it scales as $\left|z_{0}\right|^{n}$. Note that for $z_{0} \notin \mathbb{R}$, the imaginary parts originating from $z_{0}$ and its conjugate $\bar{z}_{0}$ exactly cancel out, but the scaling is still given by $\left|z_{0}\right|^{n}=e^{n \log \left|z_{0}\right|}$, i.e., $\xi=-1 / \log \left|z_{0}\right|$ is the corresponding correlation length.

If $z_{0}$ is a simple zero of $g(z)$, we have to integrate around the branch cut. Assume first that the cut goes to zero in a straight line, and consider a contour with distance $\varepsilon$ to the slit. Both the contribution from the $\varepsilon$ region around zero and the $\varepsilon$ semicircle at $z_{0}$ vanish as $\varepsilon \rightarrow 0$, and the total integral is therefore given by twice the integral along the cut,

$$
\frac{1}{\pi i} \int_{0}^{z_{0}} \frac{z^{n-1}}{\sqrt{z-z_{0}} \sqrt{h(z)}} \mathrm{d} z
$$

where again $h(z)=g(z) /\left(z-z_{0}\right)$. Intuitively, for growing $n$ the part of the integral close to $z_{0}$ becomes more and more dominating, i.e., the integral is well approximated by the modified integral where $h(z)$ has been replaced by $h\left(z_{0}\right)$. After rotating it onto the real axis, this integral - up to a phase - reads

$$
\frac{1}{\pi \sqrt{\left|h\left(z_{0}\right)\right|}} \int_{0}^{\left|z_{0}\right|} \frac{r^{n-1}}{\sqrt{\left|z_{0}\right|-r}} \mathrm{~d} r=\frac{\left|z_{0}\right|^{n-1 / 2} \Gamma(n)}{\sqrt{\pi\left|h\left(z_{0}\right)\right|} \Gamma\left(n+\frac{1}{2}\right)}
$$

which for large $n$ is

$$
\frac{1}{\sqrt{\pi\left|z_{0} h\left(z_{0}\right)\right|}} \frac{\left|z_{0}\right|^{n}}{\sqrt{n}}+O\left(\frac{\left|z_{0}\right|^{n}}{n^{3 / 2}}\right) .
$$

In order to justify the approximation $h(z) \rightsquigarrow h\left(z_{0}\right)$, consider the difference of the two respective integrals. It is bounded by

$$
|\int_{0}^{z_{0}} \frac{|z|^{n-1}}{\sqrt{\left|z-z_{0}\right|}} \underbrace{\left|\frac{1}{\sqrt{h(z)}}-\frac{1}{\sqrt{h\left(z_{0}\right)}}\right|}_{(*)} \mathrm{d} z| .
$$

On $\left[z_{0} / 2, z_{0}\right], h(z)$ is analytic and has no zeros, thus, $\left|h(z)^{-1 / 2}-h\left(z_{0}\right)^{-1 / 2}\right|<C\left|z-z_{0}\right|$, where $C$ is the maximum of the derivative of $h(z)^{-1 / 2}$ on $\left[z_{0} / 2, z_{0}\right]$. On $\left[0, z_{0} / 2\right]$, the same bound is obtained by choosing $C$ the supremum of $\left|h(z)^{-1 / 2}-h\left(z_{0}\right)^{-1 / 2}\right| /\left|z_{0} / 2\right|$ on $\left[0, z_{0} / 2\right]$. Together, $(*) \leq C\left|z-z_{0}\right|$, and the above integral is bounded by

$$
C \int_{0}^{\left|z_{0}\right|} r^{n-1} \sqrt{\left|z_{0}\right|-r} \mathrm{~d} r=C \frac{\sqrt{\pi}\left|z_{0}\right|^{n+1 / 2} \Gamma(n)}{2 \Gamma\left(n+\frac{3}{2}\right)}=O\left(\frac{\left|z_{0}\right|^{n}}{n^{3 / 2}}\right),
$$

i.e., it vanishes by $1 / n$ faster than the asymptotics derived in Eq. (25), which justifies fixing $h(z)$ at $h\left(z_{0}\right)$.

From Eq. (25), it follows that the scaling is $e^{-n / \xi} / \sqrt{n}$, where the correlation length is again $\xi=-1 / \log \left|z_{0}\right|$. The same scaling behavior can be shown to hold for appropriately chosen curved branch cuts from $z_{0}$ to 0 by relating the curved to a straight integral.

The situation gets more complicated if zeros of odd order $>1$ appear. In order to get an estimate which holds in all scenarios, we apply Cauchy's theorem to contract the unit circle in the integration (23) to a circle of radius $r>\left|z_{0}\right|$, where $z_{0}$ is the largest zero inside the unit circle. Then, the integrand can be bounded by $C_{r} r^{n-1}$ (where $C_{r}<\infty$ is the supremum of $1 / \sqrt{g}$ on the circle), and this gives a bound $2 \pi C_{r} r^{n-1}$ for the integral. This holds for all $r>\left|z_{0}\right|$, i.e., the correlation decay faster than $e^{n \log r}$ for all $r>\left|z_{0}\right|$. This does not imply that the correlations decay as $e^{n \log \left|z_{0}\right|}$, but it is still reasonable to define $-1 / \log \left|z_{0}\right|$ as the correlation length.

Theorem 10 Consider a $1 D$ chain together with a family of Hamiltonians $H(\Delta)$ with gap $\Delta>0$, where $H(\Delta)$ is continuous for $\Delta \rightarrow 0$ in the sense that all entries of $H$ converge. Then, the ground state correlations scale exponentially, and for sufficiently small $\Delta$ the correlation length is

$$
\xi \simeq \frac{1}{\sqrt{\Delta m^{*}}}
$$


Here, $m^{*}=\left(\left.\frac{\mathrm{d}^{2} \mathcal{E}(\phi)}{\mathrm{d} \phi^{2}}\right|_{\phi=\phi_{\Delta}}\right)^{-1}$ is the effective mass at the band gap.

For the discretized Klein-Gordon field (11), for example, we have $\Delta=\sqrt{1-|\kappa|}$, $m^{*}=2 \sqrt{1-|\kappa|} /|\kappa|$, and for small $\Delta$ (corresponding to $|\kappa|$ close to 1 ), one obtains $\xi \simeq \sqrt{|\kappa| / 2(1-|\kappa|)} \simeq 1 / \sqrt{2} \Delta$. Hence, the $\xi \propto 1 / \Delta$ law holds if the coupling is increased relative to the on-site energy (in which case $m^{*} \propto \Delta$ ).

More generally, if we expand the spectral function [Eq. (16)] around the band gap we are generically ${ }^{3}$ led to the dispersion relation $\mathcal{E}(k) \simeq \sqrt{\Delta^{2}+v^{2} k^{2}}(k \equiv \phi)$. By the definition of the effective mass and Theorem 10 this leads exactly to the folk theorem

$$
\xi \simeq \frac{v}{\Delta}
$$

Proof According to Theorem 9] what remains to be done is to determine the position of the largest zero $\tilde{z}$ of $g$ in the unit circle. Due to the restriction on $H(\Delta)$, the coefficients of the polynomial $g(z) z^{L}$ and thus also the zeros of $g$ continuously depend on $\Delta$, i.e., for sufficiently small $\Delta$, the zero closest to the unit circle is the one closest to the gap. In order to determine the position of this zero, we will expand $g$ around the gap. We only discuss the generic case where the gap appears only for one angle $\phi_{0}, g\left(\phi_{0}\right)=\Delta$. In the case of multiple occurrences of the gap in the spectrum, one will pick the gap which gives the zero closest to the unit circle, i.e., the largest correlation length. Furthermore, we assume $\phi_{0}=0$ without loss of generality. Otherwise, one considers $g\left(z e^{-i \phi_{0}}\right)$ instead of $g(z)$, which on the unit circle coincides with the (rotated) spectrum.

The knowledge on $g=: u+i v($ with $u, v: \mathbb{C} \rightarrow \mathbb{R}$ ) which will be used in the proof is

$$
\begin{array}{rlrl}
u(1) & =\Delta^{2} & v(1) & =0 \\
u_{\phi}(1) & =0 & v_{\phi}(1) & =0 \\
u_{\phi \phi}(1) & =2 \Delta / m^{*}>0 & v_{\phi \phi}(1) & =0
\end{array}
$$

where the subscripts denote the partial derivative with respect to the respective subscript (in Euclidean coordinates $z \equiv x+i y$, in polar coordinates $z \equiv r e^{i \phi}$ ). Note that $z=1$ is the point where the gap appears, and that $g\left(e^{i \phi}\right)=\mathcal{E}(\phi)^{2}$ is real. Therefore, the derivatives of the imaginary part $v$ along the circle vanish, while the derivatives of the real part $u$ are found to be $u(1)=\mathcal{E}(0)^{2}=\Delta^{2}, u_{\phi}(1)=2 \mathcal{E}(0) \mathcal{E}^{\prime}(0)=0$, and $u_{\phi \phi}(0)=2 \mathcal{E}^{\prime}(0)^{2}+2 \mathcal{E}(0) \mathcal{E}^{\prime \prime}(0)=$ $2 \Delta / m^{*}$, where $m^{*}=1 / \mathcal{E}^{\prime \prime}(\phi)$ is the effective mass at the band gap.

We need to exploit the relation between Euclidean and polar coordinates,

$$
\begin{array}{ll}
g_{x}(1)=g_{r}(1) & ; \quad g_{y}(1)=g_{\phi}(1) \\
g_{x x}(1)=g_{r r}(1) & ; \quad g_{y y}(1)=g_{\phi \phi}(1)+g_{r}(1)
\end{array}
$$

and the Cauchy-Riemann equations $u_{x}=v_{y}, u_{y}=-v_{x}$, and $g_{x x}+g_{y y}=0$, which together with the information (27) lead to

$$
\begin{gathered}
u(1)=\Delta^{2} ; v(1)=0 ; \\
u_{x}(1)=u_{y}(1)=v_{x}(1)=v_{y}(1)=0 ; \\
u_{x x}(1)=-2 \Delta / m^{*} ; u_{y y}(1)=2 \Delta / m^{*} ; \\
v_{x x}(1)=0 ; v_{y y}(1)=0 .
\end{gathered}
$$

Note that it is not possible to derive information about the mixed second derivates using only the information (27). However, as long as $v_{x y}$ does not vanish at $1, v$ will only stay

\footnotetext{
3 This makes the natural assumption that the minimum under the square root is quadratic. In fact, if it is of higher order, then $m^{*}=\infty$ and thus $\xi=0$, which is consistent with the findings of the following section. An example of such a behavior is given by so called 'quadratic interactions' [2] for which $H=V \oplus \mathbb{1}$, where $V$ is the square of a banded matrix.
} 
zero in direction of $x$ or $y$, but not diagonally. Since $\Delta^{2}>0$ and $2 \Delta / m^{*}>0$, the closest zero is - to second order - approximately located along the $x$ axis. By intersecting with the parabola $\Delta^{2}-\frac{\Delta}{m^{*}}(x-1)^{2}$, one finds that the zero is located at $x_{0} \approx 1-\sqrt{\Delta m^{*}}$. For small $\Delta$, the correlations thus decay with correlation length $\xi \approx-1 / \log \left(1-\sqrt{\Delta m^{*}}\right) \approx 1 / \sqrt{\Delta m^{*}}$.

\section{CRITICAL SYSTEMS}

In the following, we discuss critical systems, i.e., systems without an energy gap, $\Delta=$ $0 .{ }^{4}$ In that case, the Hamiltonian will get singular and some entries of the ground state covariance matrix will diverge, which leads to difficulties and ambiguities in the description of the asymptotic behavior of correlations. We will therefore restrict to Hamiltonians of the type

$$
H=V \oplus \mathbb{1},
$$

for which the ground state $\mathrm{CM}$ is $\gamma=V^{-1 / 2} \oplus V^{1 / 2}$. While the $Q$ part diverges, the entries of the $P$-block stay finite. Following Thm. 1(b) the extension to interactions of the form $H=H_{Q} \oplus H_{P}$ is straight forward.

In order to compute the correlations we have to determine the asymptotics of $V^{1 / 2}$, i.e.,

$$
\left(V^{1 / 2}\right)_{n}=\frac{1}{(2 \pi)^{d}} \int_{\mathcal{T}^{d}} \sqrt{\hat{V}(\phi)} e^{i n \phi} \mathrm{d} \phi .
$$

We will restrict to the cases in which the excitation spectrum $\mathcal{E}=\sqrt{\hat{V}}$ has only a finite number of zeros, i.e., finitely many points of criticality. In addition, we will also consider the special case in which the Hamiltonian exhibits a tensor product structure.

We proceed as follows. First, we consider one-dimensional critical chains and show that the correlations decay typically as $O\left(n^{-2}\right)$ and characterize those special cases where the correlations decay more rapidly. The practically important case of exactly cubic decaying interactions will be investigated in greater detail. Depending on the sign of the interaction this case will lead to a logarithmic deviation from the $n^{-2}$ behavior. Then, we turn to higher dimensional systems and show that generically the correlations decay as $n^{-(d+1)} \log n$, where $d$ is the spatial dimension of the lattice.

\section{A. One dimension}

First, we prove a Lemma which shows that although taking the square root of a smooth function destroys its differentiability, the derivatives will stay bounded.

Lemma 11 Let $f \in \mathscr{C}^{m}([-1 ; 1]), f(x) \geq 0$ with the only zero at $x=0$, and let $2 \nu \leq m$ be the order of the minimum at $x=0$, i.e., $f^{(k)}(0)=0 \forall k<2 \nu, f^{(2 \nu)}(0)>0$.

Define $g(x):=\sqrt{f(x)}$. Then, the following holds:

- For odd $\nu, g \in \mathscr{C}^{\nu-1}([-1 ; 1])$, and $g \in \mathscr{C}^{m-\nu}([-1 ; 0]), g \in \mathscr{C}^{m-\nu}([0 ; 1])$, i.e., the first $m-\nu$ derivatives (for $x \neq 0$ ) are bounded.

- For even $\nu, g \in \mathscr{C}^{m-\nu}([-1 ; 1])$.

\footnotetext{
${ }^{4}$ Note that there are different meanings of the notion criticality referring either to a vanishing energy gap or to an algebraic decay of correlations. In this section we discuss in which cases these two properties are equivalent.
} 
Proof Using the Taylor expansion $f(x)=\sum_{k=2 \nu}^{m} c_{k} x^{k}+\rho(x), \rho^{(k)}(x)=o\left(x^{m-k}\right)$ for $k \leq m$, we express $g$ as $g(x)=(\operatorname{sgn} x)^{\nu} x^{\nu} r(x)$ with

$$
r(x)=\sqrt{\sum_{k=2 \nu}^{m} c_{k} x^{k-2 \nu}+\frac{\rho(x)}{x^{2 \nu}}},
$$

where we used that $(\operatorname{sgn} x)^{\nu} x^{\nu}=\sqrt{x^{2 \nu}}$. Let us now consider the derivatives of $r(x)$. While the sum leads to a $O(1)$ contribution, the $k$ 'th derivative of the remainder behaves as $o(1) / x^{2 \nu-m+k}$. Together, this leads to

$$
\begin{array}{ll}
r^{(k)}(x)=O(1) & 2 \nu-m+k \leq 0, \\
r^{(k)}(x)=o(1) / x^{2 \nu-m+k} & 2 \nu-m+k \geq 1 .
\end{array}
$$

Now consider the $k$ 'th derivative of $g(x)$ for $x \neq 0$,

$$
g^{(k)}(x)=(\operatorname{sgn} x)^{\nu} \sum_{l=0}^{k} \underbrace{\left(\begin{array}{l}
k \\
l
\end{array}\right)\left[\frac{\mathrm{d}^{l}}{\mathrm{~d} x^{l}} x^{\nu}\right] r^{(k-l)}(x)}_{s_{l}} .
$$

Assume first $k \leq \nu$. Then, $s_{l} \propto O(1) x^{\nu-l}$ for $2 \nu-m+k-l \leq 0$, and $s_{l} \propto o(1) x^{m-\nu-k}$ for $2 \nu-m+k-l \geq 1$, and as $m \geq 2 \nu$, it follows that $g^{(k)}=O(x)$ for $k<\nu$, which cancels the discontinuity originating from $\operatorname{sgn} x$. For $k=\nu$, on the contrary, $s_{k}=O(1)$, and $\operatorname{sgn} x$ introduces a discontinuity on $g^{(k)}$, yet, it remains bounded and piecewise differentiable on $[-1 ; 0]$ and $[0 ; 1]$. The first non-bounded $s_{l}$ is found as soon as $m-\nu-k=-1$, and $g \in \mathscr{C}^{m-\nu}([0 ; 1])$ directly follows.

This also implies that for $m-\nu-k \geq 0, g(x) /(\operatorname{sgn} x)^{\nu} \in \mathscr{C}^{m-\nu}([-1 ; 1])$, i.e., the discontinuity is only due to $(\operatorname{sgn} x)^{\nu}$. Since, however, this is only discontinuous for odd $\nu$, it follows that $g \in \mathscr{C}^{m-\nu}([-1 ; 1])$ if $\nu$ even.

Theorem 12 Consider a one-dimensional critical chain with Hamiltonian $H=V \oplus \mathbb{1}$, where $V_{n}=O\left(n^{-\alpha}\right), \alpha>4$ and where $\hat{V}$ has a finite number of critical points which are all quadratic minima of $\hat{V}$. Then, $\left(\gamma_{P}\right)_{n}=O^{*}\left(n^{-2}\right)$. For $V_{n} \propto n^{-\alpha}, \alpha>3$ it even follows that $\left(\gamma_{P}\right)=\Theta\left(n^{-2}\right)$.

Note that for $V_{n} \propto n^{-\alpha}$, the extrema of $\hat{V}$ are always quadratic.

Proof We want to estimate

$$
\left(V^{1 / 2}\right)_{n}=\frac{1}{2 \pi} \int_{\mathcal{S}^{1}} g(\phi) e^{i n \phi} \mathrm{d} \phi
$$

where $g=\hat{V}^{1 / 2}$. Under both assumptions, $\hat{V} \in \mathscr{C}^{2}\left(\mathcal{S}^{1}\right)$, and all critical points are minima of order 2. It follows from Lemma 11 that $g$ is continuous with bounded derivative. Therefore, we can integrate by parts, the bracket vanishes, and we obtain

$$
\left(V^{1 / 2}\right)_{n}=-\frac{1}{2 \pi i n} \int_{0}^{2 \pi} g^{\prime}(\phi) e^{i n \phi} \mathrm{d} \phi .
$$

Now, split $\mathcal{S}^{1}$ at the zeros of $g$ into closed intervals $\mathcal{I}_{j}, \bigcup_{j} \mathcal{I}_{j}=\mathcal{S}^{1}$, and rewrite the above integral as a sum of integrals over $\mathcal{I}_{j}$. As $g^{\prime} \in \mathscr{C}\left(\mathcal{I}_{j}\right)$ (and differentiable on the inner of $\mathcal{I}_{j}$ ), one can once more integrate by parts which yields

$$
\left(V^{1 / 2}\right)_{n}=-\frac{1}{2 \pi(i n)^{2}} \sum_{j}\left(\left[g^{\prime}(\phi) e^{i n \phi}\right]_{\mathcal{I}_{j}}-\int_{\mathcal{I}_{j}} g^{\prime \prime}(\phi) e^{i n \phi} \mathrm{d} \phi\right) .
$$

Neither of the terms will vanish, but since $g^{\prime} \in \mathscr{C}\left(\mathcal{I}_{j}\right)$, the bracket is bounded. In case $V_{n} \in O\left(n^{-\alpha}\right), \alpha>4$, we have $\hat{V} \in \mathscr{C}^{3}\left(\mathcal{S}^{1}\right)$, therefore $g^{\prime \prime}$ is bounded (Lemma 11), and 
the integrals vanish as $o(1)$. Unless the contributions of the brackets for the different $\mathcal{I}_{j}$ cancel out, the $n^{-2}$ bound is tight, $\left(V^{1 / 2}\right)_{n}=O^{*}\left(n^{-2}\right)$. The tightness of the bound is also illustrated by the example which follows the proof.

For the case of an exactly polynomial decay, we additionally have to show that $g^{\prime \prime}$ is absolutely integrable for $3<\alpha \leq 4$. Then, the exactness of the bound holds because the bracket in Eq. (29) does not oscillate (the critical point is either at $\phi=0$ or at $\phi=\pi$ ), and because the integral is $o(1)$ for $g^{\prime \prime} \in \mathcal{L}^{1}\left(\mathcal{S}^{1}\right)$. In case the critical point is at $\phi=\pi$, the latter holds since $\hat{V} \in \mathscr{C}^{\infty}((0 ; 2 \pi))$ implies that $g^{\prime \prime}$ is bounded at $\pi$, and $\hat{V} \in \mathscr{C}^{2}\left(\mathcal{S}^{1}\right)$ that $g \in \mathscr{C}^{2}((-\pi, \pi))$, which together proves that $g^{\prime \prime}$ is bounded on $\mathcal{S}^{1}$.

In case the critical point is at $\phi=0$, the situation is more involved (and for $\alpha=3$, a logarithmic correction appears, cf. Theorem [14). Since $\hat{V}^{(3)}(\phi)=-\operatorname{Im}\left[\operatorname{Li}_{\alpha-3}\left(e^{i \phi}\right)\right]=$ $O\left(\phi^{\alpha-4}\right)$, we have

$$
\hat{V}^{\prime \prime}(\phi)=\hat{V}^{\prime \prime}(0)+O\left(\phi^{\alpha-3}\right), \hat{V}^{\prime}(\phi)=\hat{V}^{\prime \prime}(0) \phi+O\left(\phi^{\alpha-2}\right), \hat{V}(\phi)=\frac{1}{2} \hat{V}^{\prime \prime}(0) \phi^{2}+O\left(\phi^{\alpha-1}\right) .
$$

With this information,

$$
g^{\prime \prime}(\phi)=\frac{2 \hat{V}(\phi) \hat{V}^{\prime \prime}(\phi)-\hat{V}^{\prime}(\phi)^{2}}{4 V(\phi)^{3 / 2}}=O\left(\phi^{\alpha-4}\right),
$$

which indeed proves that $g^{\prime \prime} \in \mathcal{L}^{1}\left(\mathcal{S}^{1}\right)$, and thus $\left(V^{1 / 2}\right)_{n}=\Theta\left(n^{-2}\right)$.

As an example, consider again the discretized Klein-Gordon field of Eq. (1) which is critical for $\kappa= \pm 1$, corresponding to $\hat{V}(\phi)=1 \mp \cos \phi$. The Fourier integral is solvable and yields $\left(\gamma_{P}\right)_{n}=-\frac{2 \sqrt{2}}{\pi} \frac{(\operatorname{sgn} \kappa)^{n}}{4 n^{2}-1}=\Theta\left(n^{-2}\right)$.

Generalizations of Theorem 12. Using Lemma 11, several generalizations for the 1D critical case can be found. In the following, we mention some of them. In all cases $H=V \oplus \mathbb{1}$ is critical.

Critical points of even order.-If $V_{n}=o\left(n^{-\infty}\right)$ and the critical points are minima of order $2 \nu, \nu$ even, the correlations decay as $\left(\gamma_{P}\right)_{n}=o\left(n^{-\infty}\right)$. This is the case, e.g., if $V=X^{2}$ with $X$ itself rapidly decaying.

Critical points of higher order.- If $\hat{V}$ has critical points of order at least $2 \nu, \nu$ odd, and $V_{n}=O\left(n^{-\alpha}\right), \alpha>2 \nu+2$, then $\left(\gamma_{P}\right)_{n}=O\left(n^{-(\nu+1)}\right)$.

Minima of different orders.-If $\hat{V}$ has minima of different orders $2 \nu_{i}$, in general the minimum with the lowest odd $\nu_{i} \equiv \nu_{1}$ will determine the asymptotics, $\left(\gamma_{P}\right)_{n}=O\left(n^{-\left(\nu_{1}+1\right)}\right)$. As $\hat{V} \in \mathscr{C}^{\left(2 \max \left\{\nu_{i}\right\}\right)}\left(\mathcal{S}^{1}\right)$ is required anyway, the piecewise differentiability of $\hat{V}^{1 / 2}$ is guaranteed.

Weaker requirements on $V$.- It is possible to ease the requirements imposed on $V$ in Theo$\operatorname{rem} 12$ to $V_{n}=O\left(n^{-\alpha}\right), \alpha>3$ or $\hat{V} \in \mathscr{C}^{2}\left(\mathcal{S}^{1}\right)$, respectively. The price one has to pay is that one gets an additional $\log$ correction as in the multidimensional critical case, Theorem 15. The method to bound $g^{\prime \prime}$ is the same which is used there to derive (39).

The above proof does not cover the case of the relevant $1 / n^{3}$ interaction, which for instance appears for the motional degrees of freedom of trapped ions. In the following, we separately discuss this case. It will turn out that the scaling will depend on the sign of the coupling: while a positive sign (corresponding to the radial degrees of freedom) again gives a $\Theta\left(\frac{1}{n^{2}}\right)$ scaling as before, for the negative sign (corresponding to the axial degree of freedom) one gets $\Theta\left(\frac{\sqrt{\log n}}{n^{2}}\right)$.

Theorem 13 Consider a critical $1 D$ chain with a $1 / n^{3}$ coupling with positive sign, i.e., $H=V \oplus \mathbb{1}, V_{n}=c / n^{3}, V_{0}=3 c \zeta(3) / 2, c>0$, with $\zeta$ the Riemann zeta function. Then, the ground state correlations scale as $\left(\gamma_{P}\right)_{n}=\Theta\left(\frac{1}{n^{2}}\right)$.

Proof We take w.l.o.g. $c=1 / 2$. For this sign of the coupling, the critical point is at $\pi$, $\hat{V}(\pi)=0$. From the proof of Lemma 7, we know that $\hat{V} \in \mathscr{C}^{1}\left(\mathcal{S}^{1}\right), \hat{V} \in \mathscr{C}^{\infty}((0 ; 2 \pi))$, and that $\hat{V}^{\prime \prime}(\phi)=\log (2 \sin (\phi / 2))$ on $(0 ; 2 \pi)$. With $g:=\hat{V}^{1 / 2}$, it follows from Lemma 11 that $g \in \mathscr{C}\left(\mathcal{S}^{1}\right), g \in \mathscr{C}^{1}([-\pi ; \pi])$, and $g \in \mathscr{C}^{\infty}((0 ; \pi]), g \in \mathscr{C}^{\infty}([-\pi ; 0))$. This means that all 
derivatives $g^{(k)}, k \geq 1$ can exhibit jumps at the critical point $\pi$ but they all remain bounded. In contrast, around $\phi=0, g^{\prime}$ is continuous but $g^{\prime \prime}$ has a log divergence.

Thus, the Fourier integral

$$
\left(V^{1 / 2}\right)_{n}=\frac{1}{2 \pi} \int_{\mathcal{S}^{1}} g(\phi) e^{i n \phi} \mathrm{d} \phi
$$

can be split at 0 and $\pi$, and then integrated by parts twice. The brackets of the first integration cancel out due to continuity of $g$, and one remains with

$$
\left(V^{1 / 2}\right)_{n}=\frac{1}{\pi(i n)^{2}}\left(\left[g^{\prime}(\phi) \cos (n \phi)\right]_{0}^{\pi}+\int_{0}^{\pi} g^{\prime \prime}(\phi) \cos (n \phi) \mathrm{d} \phi\right)
$$

where we used the symmetry of $g$. One finds $\left[g^{\prime}(\phi) \cos (n \phi)\right]_{0}^{\pi}=-\sqrt{\frac{\log 2}{2}}(-1)^{n}$, and since $g^{\prime \prime}$ is integrable, the integral is $o(1)$ due to the Riemann-Lebesgue lemma. Together, this proves $\left(\gamma_{P}\right)_{n}=\Theta\left(\frac{1}{n^{2}}\right)$.

Theorem 14 Consider a critical $1 D$ chain with a $1 / n^{3}$ coupling with negative sign, i.e., $H=V \oplus \mathbb{1}, V_{n}=-c / n^{3}, V_{0}=2 c \zeta(3), c>0$, with $\zeta$ the Riemann zeta function. Then, the ground state correlations scale as $\left(\gamma_{P}\right)_{n}=\Theta\left(\frac{\sqrt{\log n}}{n^{2}}\right)$.

Proof Again, take w.l.o.g. $c=1 / 2$. For the negative sign of the interaction, the critical point is at $\phi=0$. Since at this point $\hat{V}^{\prime \prime}$ diverges, Lemma 11 cannot be applied, and the situation gets more involved.

As in the previous proof, we use that $\hat{V} \in \mathscr{C}^{1}\left(\mathcal{S}^{1}\right), \hat{V} \in \mathscr{C}^{\infty}((0 ; 2 \pi))$, and thus $\hat{V}^{1 / 2} \in$ $\mathscr{C}\left(\mathcal{S}^{1}\right), \hat{V}^{1 / 2} \in \mathscr{C}^{\infty}((0 ; 2 \pi))$. Further, $\hat{V}^{\prime \prime}(\phi)=-\log (2 \sin (\phi / 2))$ on $(0 ; 2 \pi)$, cf. the proof of Lemma 7 and with $\sin x=x\left(1+O\left(x^{2}\right)\right)$ we have $\hat{V}^{\prime \prime}(\phi)=-\log (\phi)+O\left(\phi^{2}\right)$ for $\phi \rightarrow 0$ (and similarly for $\phi \rightarrow 2 \pi)$, and therefore

$$
\hat{V}^{\prime}(\phi)=\phi(1-\log \phi)+O\left(\phi^{3}\right), \quad \hat{V}(\phi)=\frac{1}{4} \phi^{2}(3-2 \log \phi)+O\left(\phi^{4}\right) .
$$

As $\hat{V}^{1 / 2} \in \mathscr{C}\left(\mathcal{S}^{1}\right)$, we can integrate by parts one time,

$$
\left(V^{1 / 2}\right)_{n}=\frac{1}{2 \pi} \int_{\mathcal{S}^{1}} \hat{V}^{1 / 2}(\phi) e^{i n \phi} \mathrm{d} \phi=\frac{1}{\pi n} \int_{0}^{\pi} g^{\prime}(\phi) \sin (n \phi) \mathrm{d} \phi
$$

where we exploited the symmetry of $\hat{V}$, and with $g:=\hat{V}^{1 / 2}$. Then, from (30),

$$
g^{\prime}(\phi)=\frac{1-\log \phi}{\sqrt{3-2 \log \phi}}+O\left(\frac{\phi^{2}}{\sqrt{|\log \phi|}}\right), g^{\prime \prime}(\phi)=\frac{-2+\log \phi}{\phi(3-2 \log \phi)^{3 / 2}}+O\left(\frac{\phi}{\sqrt{|\log \phi|}}\right)
$$

and after another round of approximation,

$$
g^{\prime}(\phi)=\frac{\sqrt{|\log \phi|}}{\sqrt{2}}+O\left(\frac{1}{\sqrt{|\log \phi|}}\right), g^{\prime \prime}(\phi)=-\frac{1}{2^{3 / 2}} \frac{1}{\phi \sqrt{|\log \phi|}}+O\left(\frac{1}{\phi|\log \phi|^{3 / 2}}\right) \text {. }
$$

This shows that the remainder $g^{\prime}(\phi)-\sqrt{|\log \phi| / 2}$ is continuous with a absolutely integrable derivative, and by integration by parts it follows that it only leads to a contribution $O(1 / n)$ in the integral (31). Thus, it remains to investigate the asymptotics of the sine Fourier coefficients of $h(\phi)=\sqrt{|\log \phi|}$. For convenience, we split the integral (31) at 1 , and $[1 ; \pi]$ only contributes with $O(1 / n)$, as $h$ is continuous with absolutely integrable derivative on $[1 ; \pi]$. On $[0 ; 1]$, we have to compute the asymptotics of

$$
\mathcal{I}=\int_{0}^{1} \sqrt{-\log \phi} \sin (n \phi) \mathrm{d} \phi .
$$


Therefore, split the integral at $1 / n$. The left integral can be bounded directly, and the right after integration by parts [cf. the treatment of Eq. (44)]. One gets

$$
\mathcal{I} \leq \int_{0}^{1 / n} \sqrt{-\log \phi} \mathrm{d} \phi+\frac{\sqrt{\log n}}{n}+\frac{1}{n} \int_{1 / n}^{1} \frac{1}{2 \phi \sqrt{-\log \phi}} \mathrm{d} \phi=O\left(\frac{\sqrt{\log n}}{n}\right) .
$$

In order to prove that this is also a lower bound for the asymptotics, it suffices to show this for the integral (32) as all other contributions vanish more quickly. To this end, split the integral (32) into single oscillations of the sine, $J_{k}=\left[\frac{2 \pi k}{n}, \frac{2 \pi(k+1)}{n}\right], k \geq 0$. As $\sqrt{-\log \phi}$ has negative slope on $(0 ; 1)$, each of the $J_{k}$ gives a positive contribution to $\mathcal{I}$, and thus we can truncate the integral at $\frac{1}{2}$,

$$
\mathcal{I} \geq \sum_{\frac{2 \pi(k+1)}{n} \leq \frac{1}{2}} \int_{J_{k}} \sqrt{-\log \phi} \sin (n \phi) \mathrm{d} \phi .
$$

On $\left[0 ; \frac{1}{2}\right], \sqrt{-\log \phi}$ has a positive curvature, and thus, each of the integrals can be estimated by linearly approximating $\sqrt{-\log \phi}$ at the middle of each $J_{k}$ but with the slope at $\frac{2 \pi(k+1)}{n}$, which gives

$$
\int_{J_{k}} \sqrt{-\log \phi} \sin (n \phi) \mathrm{d} \phi \geq \frac{\pi}{n^{2}} \frac{1}{\frac{2 \pi(k+1)}{n} \sqrt{-\log \left[\frac{2 \pi(k+1)}{n}\right]}} .
$$

Now, we plug this into the sum (33) and bound the sum by the integral from $\frac{2 \pi}{n}$ to $\frac{1}{2}$ (the integrand in monotonically decreasing), which indeed gives a lower bound $\frac{1}{n}\left(\sqrt{\log \frac{n}{2 \pi}}-\right.$ $\sqrt{\log 2})$ on $\mathcal{I}$ and thus proves the $\Theta\left(\sqrt{\log n} / n^{2}\right)$ scaling.

\section{B. Higher dimensions}

For more than one dimension, the situation is more involved. First of all, it is clear by taking many uncoupled copies of the one-dimensional chain that there exist cases where the correlations will decay as $n^{-2}$. However, these are very special examples corresponding to Hamiltonians with a tensor product structure $H_{i_{1} i_{2}, j_{1} j_{2}}=H_{i_{1}, j_{1}} H_{i_{2}, j_{2}}^{\prime}$. In contrast, we show that for generic systems the correlations in the critical case decay as $O\left(n^{-(d+1)} \log n\right)$, where $d$ is the dimension of the lattice. The requirement is again that the energy spectrum $\mathcal{E}(\phi)$ has only a finite number of zeroes, i.e., finitely many critical points.

Note that the case of a Hamiltonian with a tensor product structure can also be solved, as in that case $\hat{V}$ becomes a product of terms depending on one $\phi_{i}$ each and thus the integral factorizes. Interestingly, although the correlations along the axes decay as $n^{-2}$, the correlations in a fixed diagonal direction will decay as $n_{1}^{-2} \cdots n_{d}^{-2} \propto\|n\|^{-2 d}$ and thus even faster than in the following theorem. The $O\left(\|n\|^{-(d+1)} \log \|n\|\right)$ decay of the theorem holds isotropically, i.e., independent of the direction of $n$.

Theorem 15 Consider a d-dimensional bosonic lattice with a critical Hamiltonian $H=$ $V \oplus \mathbb{1 1}$. Then the P-correlations of the ground state decay as

$$
O\left(\|n\|^{-(d+1)} \log \|n\|\right)
$$

if the following holds: $\hat{V} \in \mathscr{C}^{d+1}$ [e.g., the correlations decay as $\left.O\left(\|n\|^{-(2 d+1+\varepsilon)}\right), \varepsilon>0\right], \hat{V}$ has only a finite number of zeros which are quadratic minima, i.e., the Hessian $\left(\frac{\partial^{2} \hat{V}(\phi)}{\partial \phi_{i} \partial \phi_{j}}\right)_{i j}$ is positive definite at all zeros.

Proof We have to evaluate the asymptotic behavior of the integral

$$
\left(\hat{V}^{1 / 2}\right)_{n}=\frac{1}{(2 \pi)^{d}} \int_{\mathcal{T}^{d}} \mathrm{~d}^{d} \phi \sqrt{\hat{V}(\phi)} \cos [n \phi] .
$$


Let us first briefly sketch the proof. We start by showing that it suffices to analyze each critical point separately. To this end, we show that is is possible to smoothly cut out some environment of each critical point which reproduces the asymptotic behavior. Then, we rotate the coordinate system such that we always look at the correlations in a fixed direction, and integrate by parts - which surprisingly can be carried out as often as $\hat{V}$ is differentiable, as all the brackets vanish. Therefore, the information about the asymptotics is contained in the remaining integral, and after a properly chosen number of partial integrations, we will attempt to estimate this term.

Let now $\zeta_{i}, i=1, \ldots, I$ be the zeros of $\hat{V}$. Clearly, these will be the only points which contribute to the asymptotics as everywhere else $\sqrt{\hat{V}}$ is $\mathscr{C}^{d+1}$. In order to separate the contributions coming from the different $\zeta_{i}$, we will make use of so-called neutralizers [27]. For our purposes, these are functions $\mathcal{N}_{\xi_{0}, r} \in \mathscr{C}^{\infty}\left(\mathbb{R}^{d} \rightarrow[0 ; 1]\right)$ which satifsy

$$
\mathcal{N}_{\xi_{0}, r}(\xi)=\left\{\begin{array}{l}
1:\left\|\xi-\xi_{0}\right\| \leq r / 2 \\
0:\left\|\xi-\xi_{0}\right\| \geq r
\end{array}\right.
$$

and are rotationally symmetric (cf. 27] for an explicit construction). For each $\zeta_{i}$, there exists an $r_{i}$ such that the balls $B_{r_{i}}\left(\zeta_{i}\right)$ do not intersect. We now define the functions

$$
f_{i}(\phi):=\sqrt{\hat{V}(\phi)} \mathcal{N}_{\zeta_{i}, r_{i}}(\phi), \quad \rho(\phi):=\sqrt{\hat{V}(\phi)}-\sum_{i=1}^{I} f_{i}(\phi) .
$$

Clearly, $\rho$ is $\mathscr{C}^{d+1}$, and so is each $f_{i}$ except at $\zeta_{i}$. Furthermore, each $f_{i}$ is still the square root of a $\mathscr{C}^{d+1}$ function. By definition,

$$
\left(\hat{V}^{-1 / 2}\right)_{n}=\frac{1}{(2 \pi)^{d}} \sum_{i=1}^{I} \int_{\mathcal{T}^{d}} \mathrm{~d}^{d} \phi f_{i}(\phi) \cos [n \phi]+\frac{1}{(2 \pi)^{d}} \int_{\mathcal{T}^{d}} \mathrm{~d}^{d} \phi \rho(\phi) \cos [n \phi],
$$

i.e., it suffices to look at the asymptotics of each $f_{i}$ separately. The contribution of $\rho$ is $O\left(\|n\|^{-(d+1)}\right)$ as can be shown by successive integrations by parts just as for the non-critial lattice (cf. the proof of Lemma 5).

Let us now analyze the integrals

$$
I_{i}=\int_{B_{r_{i}}\left(\zeta_{i}\right)} \mathrm{d}^{d} \phi f_{i}(\phi) \cos [n \phi] .
$$

The integration range can be restriced to $B_{r_{i}}\left(\zeta_{i}\right)$ as $f_{i}$ vanishes outside the ball. By a rotation, this can be mapped to an integral where $n=(\|n\|, 0, \ldots, 0)$, whereas $f_{i}$ is rotated to another function $\tilde{f}_{i}$ with the same properties,

$$
I_{i}=\int_{B_{r_{i}}\left(\zeta_{i}\right)} \mathrm{d}^{d} \phi \tilde{f}_{i}(\phi) \cos \left[\|n\| \phi_{1}\right]
$$

Since the integrand is continuous and thus bounded, it is absolutely integrable, and from Fubini's theorem, one finds

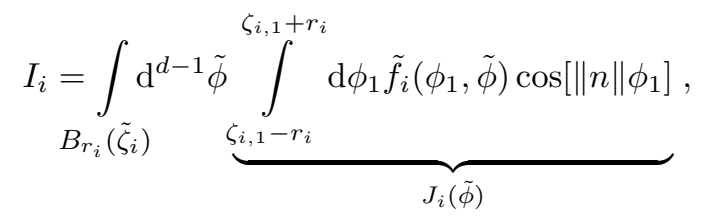

where we separated out the integration over the first component. The vector $\tilde{\phi}$ denotes the components $2 \ldots d$ of $\phi$. The extension of the integration range to a cylinder is possible as $\tilde{f}_{i}$ vanishes outside $B_{r_{i}}\left(\zeta_{i}\right)$.

Let us now require $\tilde{\phi} \neq \tilde{\zeta}_{i}$. This does not change the integral since the excluded set is of measure zero, but it ensures that $\tilde{f}_{i}$ is in $\mathscr{C}^{d+1}$. This allows us to integrate the inner integral $J_{i}(\tilde{\phi})$ by parts up to $d+1$ times, and each of the brackets

$$
\left[\tilde{f}_{i}^{(k)}\left(\phi_{1}, \tilde{\phi}\right) \frac{1}{\|n\|^{k}} \cos \left(\|n\| \phi_{1}-k \pi / 2\right)\right]_{\phi_{1}=\zeta_{i, 1}-r_{i}}^{\zeta_{i, 1}+r_{i}}
$$


appearing in the $k^{\prime}$ th integration step vanishes. Here, $\tilde{f}_{i}^{(d)}\left(\phi_{1}, \tilde{\phi}\right)=\partial^{d} \tilde{f}_{i}\left(\phi_{1}, \tilde{\phi}\right) / \partial \phi_{1}^{d}$ is the $d$ 'th partial derivative with respect to the first argument. After integrating by parts $d$ times, we obtain

$$
I_{i}=\frac{1}{\|n\|^{d}} \int_{B_{r_{i}}\left(\tilde{\zeta}_{i}\right)} \mathrm{d}^{d-1} \tilde{\phi} \int_{\zeta_{i, 1}-r_{i}}^{\zeta_{i, 1}+r_{i}} \mathrm{~d} \phi_{1} \tilde{f}_{i}^{(d)}\left(\phi_{1}, \tilde{\phi}\right) \cos \left[\|n\| \phi_{1}-d \pi / 2\right] .
$$

Now we proceed as follows: first, we show that the order of integration can be interchanged, and second, we show that for the function obtained after integrating $\tilde{f}_{i}^{(d)}$ over $\tilde{\phi}$, the Fourier coefficients vanish as $\log (\|n\|) /\|n\|$.

The central issue for what follows is to find suitable bounds on $\left|\tilde{f}_{i}^{(k)}\right|$. Therefore, define $\tilde{f}_{i}^{2}=: h_{i} \in \mathscr{C}^{d+1}$. By virtue of Taylor's theorem, and as $h_{i}\left(\zeta_{i}\right)=0$ is a minimum,

$$
h_{i}(\phi)=\frac{1}{2}\left(\phi-\zeta_{i}\right) \cdot\left(\mathbf{D}^{2} h_{i}\left(\zeta_{i}\right)\right)\left(\phi-\zeta_{i}\right)+o\left(\left\|\phi-\zeta_{i}\right\|^{2}\right)
$$

with $\mathbf{D}^{2}$ the second derivative. As the first term is bounded by $\frac{1}{2}\left\|\mathbf{D}^{2} h_{i}\left(\zeta_{i}\right)\right\|_{\infty}\left\|\phi-\zeta_{i}\right\|^{2}$ and the second vanished faster than $\left\|\phi-\zeta_{i}\right\|^{2}$, we can find $\varepsilon_{i}>0$ and $C_{1}>0$ such that

$$
\left|h_{i}(\phi)\right| \leq C_{1}\left\|\phi-\zeta_{i}\right\|^{2} \quad \forall\left\|\phi-\zeta_{i}\right\|<\varepsilon_{i} .
$$

By looking at the Tayor series of $h_{i}^{\prime} \equiv \partial h_{i} / \partial \phi_{1}$ up to the first order we also find that there are $\varepsilon_{i}>0$ and $C_{2}>0$ such that

$$
\left|h_{i}^{\prime}(\phi)\right| \leq C_{2}\left\|\phi-\zeta_{i}\right\| \quad \forall\left\|\phi-\zeta_{i}\right\|<\varepsilon_{i}
$$

In addition to these upper bounds, we will also need a lower bound on $\left|h_{i}\right|$. Again, by the Taylor expansion of $h_{i}$ around $\zeta_{i}$, we find

$$
\left|h_{i}(\phi)\right| \geq \lambda_{\min }\left[\mathbf{D}^{2} h_{i}\left(\zeta_{i}\right)\right]-o\left(\left\|\phi-\zeta_{i}\right\|^{2}\right),
$$

and as all the zeros are quadratic minima, i.e., $\lambda_{\min }\left[\mathbf{D}^{\mathbf{2}} h_{i}\left(\zeta_{i}\right)\right]>0$, there exist $\varepsilon_{i}>0$, $C_{3}>0$ such that

$$
\left|h_{i}(\phi)\right| \geq C_{3}\left\|\phi-\zeta_{i}\right\|^{2} \quad \forall\left\|\phi-\zeta_{i}\right\|<\varepsilon_{i}
$$

Clearly, $\varepsilon_{i}$ can be chosen equal in Eqs. (36]38). Note that the bounds can be chosen to be invariant under rotation of $h_{i}$ and thus of $\tilde{f}_{i}$. This holds in particular for the $\varepsilon_{i}$ as the remainders of Taylor series vanish uniformly. Thus, the bound we will obtain for the correlation function indeed only depends on $\|n\|$ and not on the direction of $n$.

Now, we use the conditions (36, 38) to derive bounds on $\left|\tilde{f}_{i}^{(k)}\right|$. Therefore, note that from $\tilde{f}_{i} \equiv \sqrt{h_{i}}$ it follows that

$$
\tilde{f}_{i}^{(k)}=\frac{\sum_{\substack{j_{1}+\cdots+j_{k}=k \\ j_{\nu}=0,1,2, \ldots}} c_{j_{1} \ldots j_{k}} h_{i}^{\left(j_{1}\right)} \cdots h_{i}^{\left(j_{k}\right)}}{h_{i}^{(2 k-1) / 2}} .
$$

One can easily check that for each term in the numerator, the number $K_{0}$ of zeroth derivatives and the number $K_{1}$ of first derivatives of $h_{i}$ satisfy $2 K_{0}+K_{1} \geq k$. By bounding all higher derivatives of $h_{i}$ from above by constants, we find that the modulus of each summand in the numerator, and thus the modulus of the numerator itself, can be bounded above by $C^{\prime}\left\|\phi-\zeta_{i}\right\|^{k}$ in the ball $B_{\varepsilon_{i}}\left(\zeta_{i}\right)$ with some $C^{\prime}>0$. On the other hand, it follows directly from (38) that the modulus of the denominator is bounded below by $C^{\prime \prime}\left\|\phi-\zeta_{i}\right\|^{2 k-1}, C^{\prime \prime}>0$, such that in total

$$
\left|\tilde{f}_{i}^{(k)}(\phi)\right| \leq C \frac{1}{\left\|\phi-\zeta_{i}\right\|^{k-1}} ; \quad 1 \leq k \leq d+1 .
$$

Note that this holds not only inside $B_{\varepsilon_{i}}\left(\zeta_{i}\right)$ but in the whole domain of $f_{i}$, as outside $B_{\varepsilon_{i}}\left(\zeta_{i}\right)$, $f_{i}$ is $\mathscr{C}^{d+1}$ and thus all the derivatives are bounded. 
Eq. (39) is the key result for the remaining part of the proof. First, it can be used to bound the integrand in (35) by an integrable singularity (this is most easily seen in spherical coordinates, where $1 / r^{d-1}$ is integrable in a $d$-dimensional space). Hence, the order of integration in (35) can be interchanged, and it remains to investigate the asymptotics of the integral

$$
\begin{aligned}
I_{i} & =\frac{1}{\|n\|_{\zeta_{i, 1}}^{d}} \int_{r_{i}}^{\zeta_{i, 1}+r_{i}} \mathrm{~d} \phi_{1} g_{i}\left(\phi_{1}\right) \cos \left[\|n\| \phi_{1}-d \pi / 2\right], \quad \text { with } \\
g_{i}\left(\phi_{1}\right) & \equiv \int_{B_{r_{i}}\left(\tilde{\zeta}_{i}\right)} \mathrm{d}^{d-1} \tilde{\phi} \tilde{f}_{i}^{(d)}\left(\phi_{1}, \tilde{\phi}\right) .
\end{aligned}
$$

From (39), we now derive bounds on $g_{i}\left(\phi_{1}\right)$ and its first derivative. Again, we may safely fix $\phi_{1} \neq \zeta_{i, 1}$ as this has measure zero. Then, using (39) we find that

$$
\left|g_{i}\left(\phi_{1}\right)\right| \leq \int_{0}^{r_{i}} \frac{C}{\left.\left(\left(\phi_{1}-\zeta_{i, 1}\right)^{2}+r^{2}\right)\right)^{(d-1) / 2}} S_{d-1} r^{d-2} \mathrm{~d} r
$$

where we have transformed into spherical coordinates $\left[S_{d-1}\right.$ is the surface of the $(d-1)$ dimensional unit sphere] and assumed the $l_{2}$-norm. Since $\left(\phi_{1}-\zeta_{1}\right)^{2}+r^{2} \geq r^{2}$, the integrand can be bounded once again, and we find

$$
\begin{aligned}
\left|g_{i}\left(\phi_{1}\right)\right| & \leq \int_{0}^{r_{i}} \frac{C S_{d-1}}{\left(\left(\phi_{1}-\zeta_{i, 1}\right)^{2}+r^{2}\right)^{1 / 2}} \mathrm{~d} r \\
& =C\left(-\log \left|\phi_{1}-\zeta_{i, 1}\right|+\log \left[r_{i}+\sqrt{r_{i}^{2}+\left(\phi_{1}-\zeta_{i, 1}\right)^{2}}\right]\right) \\
& \leq-C \log \left|\phi_{1}-\zeta_{i, 1}\right|
\end{aligned}
$$

where in the last step we used that in (40) $\left|\phi_{1}-\zeta_{i, 1}\right|<r_{i}$ and that $r_{i}$ can be chosen sufficiently small.

Next, we derive a bound on $g_{i}^{\prime}\left(\phi_{1}\right)$. As we fix $\phi_{1} \neq \zeta_{1}$, the integrand in (41) is $\mathscr{C}^{1}$ and we can take the differentiation into the integral,

$$
g_{i}^{\prime}\left(\phi_{1}\right)=\int_{B_{r_{i}}\left(\tilde{\zeta}_{i}\right)} \mathrm{d}^{d-1} \tilde{\phi} \tilde{f}_{i}^{(d+1)}\left(\phi_{1}, \tilde{\phi}\right) .
$$

Again, we bound the integrand by virtue of Eq. (39) and obtain

$$
\begin{aligned}
\left|g_{i}^{\prime}\left(\phi_{1}\right)\right| & \leq \int_{0}^{r_{i}} \frac{C S_{d-1}}{\left(\left(\phi_{1}-\zeta_{i, 1}\right)^{2}+r^{2}\right)} \mathrm{d} r \\
& =C \frac{\arctan \left[\frac{r_{i}}{\left|\phi_{1}-\zeta_{i, 1}\right|}\right]}{\left|\phi_{1}-\zeta_{i, 1}\right|} \leq \frac{C^{\prime}}{\left|\phi_{1}-\zeta_{i, 1}\right|} .
\end{aligned}
$$

Finally, these two bounds will allow us to estimate (40) and thus the asymptotics of the correlations in the lattice. We consider one half of the integral (40),

$$
\int_{\zeta_{i, 1}}^{\zeta_{i, 1}+r_{i}} \mathrm{~d} \phi_{1} g_{i}\left(\phi_{1}\right) \cos \left[\|n\| \phi_{1}-d \pi / 2\right],
$$

as both halves contribute equally to the asymptotics. We then split the integral at $\zeta_{i, 1}+$ $r_{i} /\|n\|$. The left part gives

$$
\begin{aligned}
& \left|\int_{\zeta_{i, 1}}^{\zeta_{i, 1}+r_{i} /\|n\|} \mathrm{d} \phi_{1} g_{i}\left(\phi_{1}\right) \cos \left[\|n\| \phi_{1}-d \pi / 2\right]\right| \stackrel{42}{\leq} \int_{\zeta_{i, 1}}^{\zeta_{i, 1}+r_{i} /\|n\|} \mathrm{d} \phi_{1}\left(-\log \left|\phi_{1}-\zeta_{i, 1}\right|\right) \\
& =\frac{r_{i}-r_{i} \log r_{i}+r_{i} \log \|n\|}{\|n\|} .
\end{aligned}
$$


The right part of the split integral (44) can be estimated by integration by parts,

$$
\begin{aligned}
& \left|\int_{\zeta_{i, 1}+r_{i} /\|n\|}^{\zeta_{i, 1}+r_{i}} \mathrm{~d} \phi_{1} g_{i}\left(\phi_{1}\right) \cos \left[\|n\| \phi_{1}-d \pi / 2\right]\right| \leq \\
& \quad \leq\left|\left[g_{i}\left(\phi_{1}\right) \frac{1}{\|n\|} \cos \left[\|n\| \phi_{1}-(d+1) \pi / 2\right]\right]_{\zeta_{i, 1}+r_{i} /\|n\|}^{\zeta_{i, 1}+r_{i}}\right|+\frac{1}{\|n\|} \int_{\zeta_{i, 1}+r_{i} /\|n\|}^{\zeta_{i, 1}+r_{i}} \mathrm{~d} \phi_{1}\left|g_{i}^{\prime}\left(\phi_{1}\right)\right| \\
& \frac{(42 \mid 43}{\leq} C \frac{\log \|n\|}{\|n\|}+C^{\prime} \frac{\left|\log r_{i}\right|}{\|n\|} .
\end{aligned}
$$

Thus, both halves [Eqs. (45), (46)] give a log $\|n\| /\|n\|$ bound for the integral (44), and thus the integral $I_{i}$ is asymptotically bounded by $\log \|n\| /\|n\|^{d+1}$ following Eq. (40). As the number of such integrals in (34) is finite, this proves that the correlations of the ground state decay at least as $\log \|n\| /\|n\|^{d+1}$.

\section{GAUSSIAN MATRIX PRODUCT STATES}

Recently, so-called Matrix Product States (MPS) have attracted growing interest in quantum information theory. These states appear in the DMRG (Density Matrix Renormalization Group) method which is a powerful tool to compute ground state properties of translational invariant Hamiltonians. From a quantum information perspective, this class of states can be given a physical interpretation in terms of projected entangled pairs: they can be obtained by taking a chain of maximally entangled pairs of dimension $D$ and applying a map $\mathcal{T}$ as in Fig. 2 in a translational invariant fashion. In the limit of large bond dimension $D$, this allows to approximate arbitrary translational invariant states. In finite dimensions, the MPS representation turned out to be a very fruitful concept as it led to new powerful numerical algorithms [28 31] accompanied by a better understanding of their efficiency [32], and new insights into renormalization group transformations [33] and sequential quantum generators [34]. In the following, we generalize matrix product states to the Gaussian scenario.

\section{A. Definition of Gaussian MPS}

We start by defining Gaussian matrix product states (GMPS). The definition resembles the physical interpretation of finite-dimensional matrix product states as projected entangled pairs (PEPs). In finite dimensions, MPS can be described by taking maximally entangled pairs of dimension $D$ between adjacent sites, and applying arbitrary local operations on each site, mapping the $D \times D$ input to a $d$-dimensional output state. Similarly, GMPS are obtained by taking a number of entangled bonds and applying local (not necessarily trace-preserving)

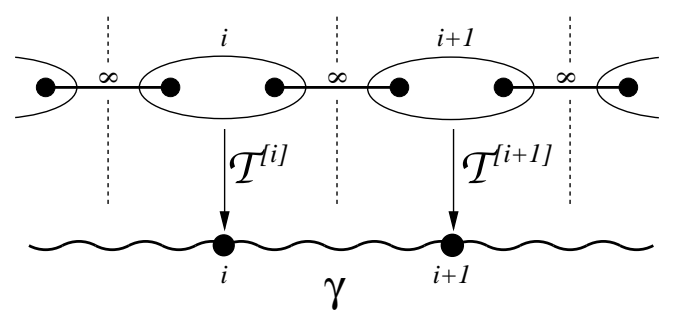

FIG. 2: Construction of Gaussian Matrix Product States (GMPS). GMPS are obtained by taking a fixed number $M$ of maximally entangled (i.e., EPR) pairs shared by adjacent sites, and applying an arbitrary $2 M$ to 1 mode Gaussian operation $\mathcal{T}^{[i]}$ on site $i$. 


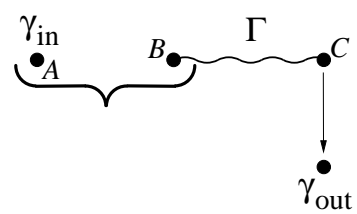

FIG. 3: The Jamiolkowski isomorphism. The Gaussian channel described by the state $\Gamma$ can be implemented by projecting the input state $\gamma_{\text {in }}$ (mode $A$ ) and the input port of $\Gamma$ (mode $B$ ) onto the EPR state (symbolized by curly brackets). In case of success, the output is obtained in mode $C$. The operation can be made trace-preserving by measuring in a basis of displaced EPR states, and displacing $C$ according to the measurement outcome.

operations $\mathcal{T}^{[i]}$, where the boundary conditions can be taken either open or closed. Any GMPS is completely described by the type of the bonds and by the operations $\mathcal{T}^{[i]}$. Note that this construction holds independent of the spatial dimension. For one dimension, it is illustrated in Fig. 2. As matrix product states are frequently used to describe translationally invariant systems, an inportant case is given if all maps are identical, $\mathcal{T}^{[i]}=\mathcal{T} \forall i$.

In order to define MPS in the Gaussian world, we have to decide on the type of the bonds as well as on the type of operations. We choose both the bonds to be Gaussian states and the operations to be Gaussian operations, i.e., operations mapping Gaussian inputs to Gaussian outputs. For now, we will take the bonds to be maximally entangled (i.e., EPR) states, such that the only parameter originating from the bonds is the number $M$ of EPRs. We show later on how the case of finitely entangled bonds can be easily embedded.

As to the operations, we will allow for arbitrary Gaussian operations. Operations of this type are most easily described by the Jamiolkowski isomorphism [35]. There, any Gaussian operation $\mathcal{T}$ which maps $N$ input modes to $M$ output modes can be described by an $N+M$ mode covariance matrix $\Gamma$ with block $B$ (input) and $C$ (output). The corresponding map on some input state $\gamma_{\text {in }}$ in mode $A$ is implemented by projecting the modes $A$ and $B$ onto an EPR state as shown in Fig. 3, such that the output state $\mathcal{T}\left(\gamma_{\text {in }}\right)$ is obtained in mode $C$. Conversely, the matrix $\Gamma$ which represents the channel $\mathcal{T}$ is obtained by applying the channel to one half of a maximally entangled state. The duality between $\mathcal{T}$ and $\Gamma$ is most easily understood in terms of teleportation, and shows that this characterization encompasses all Gaussian operations. Note that the protocol of Fig. [3] can be always made trace-preserving by projecting onto the set of phase-space displaced EPR states and correcting the displacement of mode $C$ according to the measurement outcome [36].

In the following, we will denote all maps $\mathcal{T}$ by their corresponding $\mathrm{CM} \Gamma$. Sometimes, we will speak of the modes $B$ and $C$ as input and output ports of $\Gamma$, respectively.

We now discuss how the covariance matrix of the output will depend on the $\mathrm{CM}$ of the input and on the channel $\Gamma$ [36, 37]. This is most easily computed in the framework of characteristic functions [38]. The characteristic function of the output is given by

$$
\chi_{C}\left(\xi_{C}\right) \propto \int e^{-\xi_{A}^{T} \gamma_{\text {in }} \xi_{A}} e^{-\xi_{B C}^{T} \Gamma \xi_{B C}} \delta\left(x_{A}-x_{B}\right) \delta\left(p_{A}+p_{B}\right) \mathrm{d} \xi_{A B},
$$

and by integrating out subsystem $A$,

$$
\chi_{C}\left(\xi_{C}\right) \propto \int e^{-\xi_{B C}^{T} M \xi_{B C}} \mathrm{~d} \xi_{B},
$$

with

$$
M=\left(\begin{array}{cc}
\theta \gamma \theta+\Gamma_{B} & \Gamma_{B C} \\
\Gamma_{C B} & \Gamma_{C}
\end{array}\right) .
$$

Basically, the integration $\int \mathrm{d} \xi_{A} \delta\left(x_{A}-x_{B}\right) \delta\left(p_{A}+p_{B}\right)$ does the following: first, it applies the partial transposition $\theta \equiv\left(\begin{array}{cc}1 & 0 \\ 0 & -1\end{array}\right)$ to one of the subsystems, and second, it collapses the two systems $A$ and $B$ in the covariance matrix by adding the corresponding entries. The integration over $\xi_{B}$, one the other hand, leads to a state whose CM is the Schur complement 
of $M_{11}, M_{22}-M_{21} M_{11}^{-1} M_{12}$, such that the output state is described by the CM

$$
\gamma_{\text {out }}=\Gamma_{C}-\Gamma_{C B} \frac{1}{\Gamma_{B}+\theta \gamma_{\mathrm{in}} \theta} \Gamma_{B C} .
$$

Let us briefly summarize how to perform projective measurements onto the EPR state in the framework of CMs, where we denote the measured modes by $A$ and $B$, while $C$ is the remaining part of the system. First, apply the partial transposition to $B$, second, collapse $A$ and $B$, and third, take the Schur complement of the collapsed mode $A B$, which gives the output CM of $C$.

As we discuss Gaussian matrix product states in connection with ground states of Hamiltonians, we are mainly interested in pure GMPS. Particularly, a GMPS is pure if the $\Gamma^{[i]}$ which describe the operations $\mathcal{T}^{[i]}$ are taken to be pure, which we assume from now on.

Let us finally emphasize that the given defintion of MPS holds independent of the spatial dimension of the system, as do most of the following results, and in fact applies to an arbitrary graph.

\section{B. Completeness of Gaussian MPS}

In the following, we show that any pure and translational invariant state can be approximated arbitrarily well by translational invariant Gaussian matrix product states, i.e., GMPS with identical local operations $\mathcal{T}$. (Without translational invariance, this is clear anyway: the complete state is prepared locally and teleported to its destination using the bonds.) The proof is presented for one dimension, but can be extended to higher spatial dimensions. For the proof, we use a theorem on the simulation of translational invariant Hamiltonians which is proven in the Appendix (Theorem 16). For our purposes, it says that in order to simulate an arbitrary translational invariant Hamiltionian with reflection symmetry, it suffices if one can implement translational invariant local and nearest neighbor Hamiltonians. ${ }^{5}$

a)

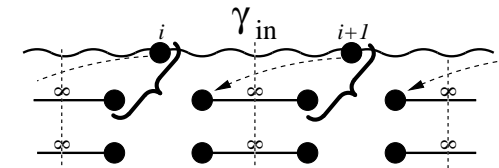

b)

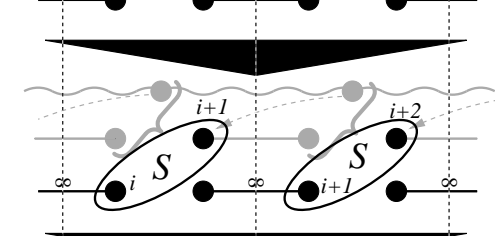

c)

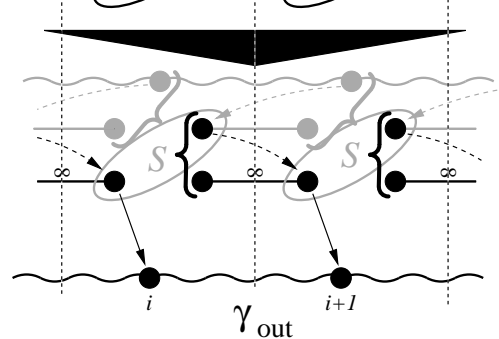

FIG. 4: Implementation of a translational invariant nearest neighbor Hamiltonian in a translational invariant fashion. Starting from $\gamma_{\text {in }}$, the input ist first teleported to the left, then, the infinitesimal time evolution $S=e^{\sigma H}, H \ll 1$, is performed, and finally, the state is teleported back.

\footnotetext{
5 This naturally extends to higher dimensions. For two dimensions, e.g., one needs nearest neighbor interactions and in addition one interaction with the closest neighbor along the diagonal in order to break the reflection symmetry. The implementation of such an interaction in the MPS formalism is a straightforward extension of the presented method.
} 
Given a translational invariant state $\gamma$, there is a translational invariant Hamiltonian $H$ which transforms the separable state $\mathbb{1}$ into $\gamma, \gamma=S S^{T}, S=e^{\sigma H}$. According to Theorem 16, this time evolution can be approximated arbitrarily well by a sequence of translational invariant local (one-mode) and nearest neighbor (two-mode) Hamiltonians $H_{j}$,

$$
e^{\sigma H} \approx \prod_{j=1}^{J} e^{\oplus_{n} \sigma H_{j}},
$$

where the $H_{j}$ act on one or two modes, respectively, and approach the identity for growing $J$.

Clearly, translational invariant local Hamiltionians can be implemented by local maps without using any EPR bonds. In the following, we show how translational invariant nearestneighbor interactions can be implemented by exploiting the entanglement of the bonds. The whole procedure is illustrated in Fig. 4 and requires two EPR pairs per site. We start with some initial state $\gamma_{\text {in }}$ onto which we want to apply $S_{\oplus}=e^{\oplus \sigma H_{j}} \approx \mathbb{1}+\bigoplus_{n} \sigma H_{j}$.

First, we perform local EPR measurements between the modes of $\gamma_{\text {in }}$ and one of the bonds in order to teleport the modes of $\gamma_{\text {in }}$ to the left, cf. Fig. 4 a. Then, the infinitesimal symplectic operation $S=e^{\sigma H_{j}}$ is applied to the left-teleported mode and the second bond, Fig. 4 $\mathrm{b}$. In the last step, another EPR measurement is performed which teleports the left-teleported mode back to the right, and "into" the mode on which the adjacent $S$ was applied. As the operations $e^{\sigma H_{j}} \approx \mathbb{1}+\sigma H_{j}$ all commute, the "nested" application of the nearest neighbor symplectic operations $S$ indeed give $S_{\oplus}$. Thus, the remaining mode indeed contains the output $\gamma_{\text {out }}=S_{\oplus} \gamma_{\text {in }} S_{\oplus}^{T}$. The whole decomposition (47) can be implemented by iterated application of the whole protocol of Fig. 4 .

\section{Gaussian MPS with finitely entangled bonds}

In this subsection we show that in general, infinitely entangled bonds can be replaced by finitely entangled ones. Intuitively, this should be possible whenever the channel $\mathcal{T}{ }^{[i]}$ destroys some of the entanglement of the bond anyway, i.e., $\Gamma^{[i]}$ is non-maximally entangled. In that case, it should be possible to use a less entangled bond while choosing a channel which does not destroy entanglement any more.

The method is illustrated in Fig. 5. Again, for reasons of clarity we restrict to one dimension and one bond. The argument however appies independent of the spatial dimension and the number of bonds. The only restriction we have to make is the restriction to pure GMPS, i.e., those with pure $\Gamma^{[i]}$.

a)

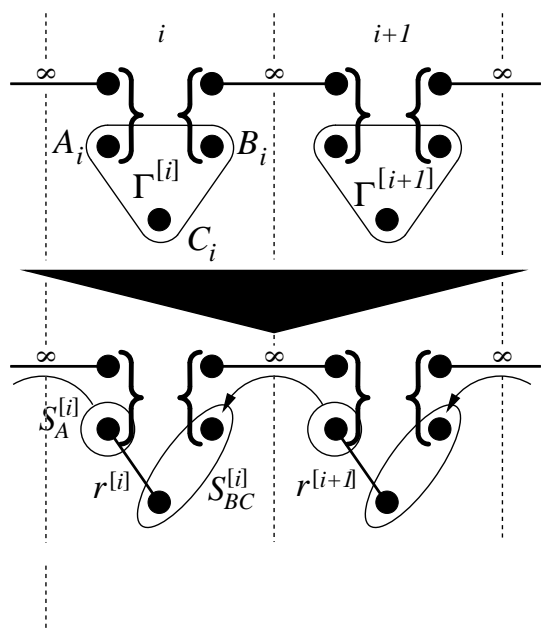

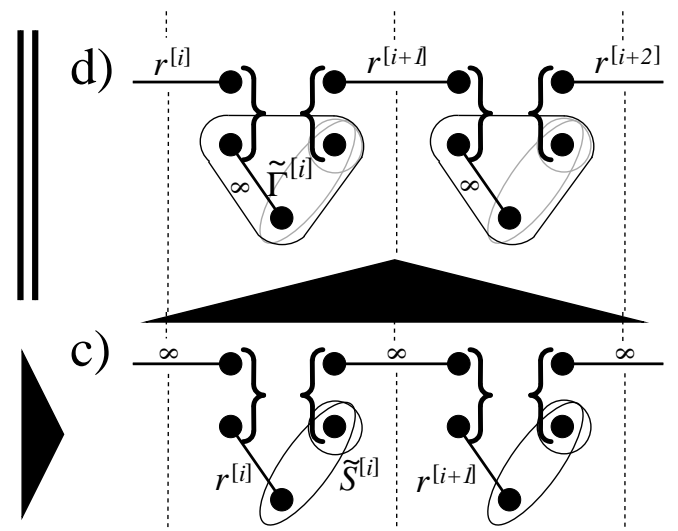

FIG. 5: How to make the bonds of GMPS finitely entangled. a) The initial MPS. b) Do a Schmidt decomposition of the original map $\Gamma$. c) Move the $S_{A}^{[i]}$ through the infinitely entangled bond to the next site. d) Swap the finitely and the infinitely entangled pair. 
Consider a GMPS with local channels given by $\Gamma^{[i]}$ and infinitely entangled bonds, Fig. 5 a . First, apply a Schmidt decomposition 39] to $\Gamma^{[i]}$ in the partition $A \mid B C$, which can be always done as long as $\Gamma^{[i]}$ is pure. The Schmidt decomposition allows us to rewrite the state as shown in Fig. $5 \mathrm{~b}$ - an entangled state between modes $A$ and $C$ with two-mode squeezing $r^{[i]}, B$ in the coherent state $\mathbb{1}$, and sympectic operations $S_{A}^{[i]}$ and $S_{B C}^{[i]}$ which are applied to modes $A$ and $B C$, respectively. As the bond itself is infinitely entangled, we can teleport the sympectic operation through the bond to the next site as indicated in Fig. 5 b. Then, $S_{A}^{[i+1]}$ can be merged with $S_{B C}^{[i]}$ to a new operation $\tilde{S}^{[i]}$ acting on modes $B$ and $C$ of site $i$ (Fig. 55). Finally, in the triples consisting of one maximally entangled state, one nonmaximally entangled state, and the projection onto the EPR state, the maximally and the non-maximally entangled state can be swapped, resulting in Fig. 5. d. There, we have finitely entangled bonds, while the infinite entanglement has been moved into the new maps $\tilde{\Gamma}^{[i]}$.

It is tempting to apply this construction to the completeness proof of the preceding section in order to obtain a construction which is less wasting with respect to resources. However, for any iterative protocol this is most likely difficult to achieve. The reason for this is found in the no-distillation theorem which states that with Gaussian operations, it is not possible to increase the amount of entanglement [36] between two parties. Particularly, this implies that in each step of an iterative protocol, the bonds need to have at least as much entanglement as can be obtained at the output of this step, maximized over all inputs where the entanglement is increased. This is indeed a severe restriction, although it does not imply the impossibility of such a protocol. One could, e.g., create a hightly entangled state in the first step and then approach the desired state by decreasing the entanglement in each step. Still, it seems most likely that a sequence of MPS which approach a given state efficiently will have to involve more and more bonds simultaneously and thus cannot be constructed in an iterative manner.

\section{Correlation functions of Gaussian MPS}

In this section, we show how to compute correlations functions from the maps $\Gamma^{[i]}$ which describe the GMPS. We show that this can be done efficiently, i.e., in polynomial time independent of the dimension of the graph which is different to the finite dimensional case. Of course, this is not too surprising as Gaussian states can be fully characterized by a number of paramaters quadratic in the number of modes.

Let us start with the general case of different $\Gamma^{[i]}$, as in Fig. 6a. The calculation can be facilitated by the simple observation that the triples consisting of two projective measure-

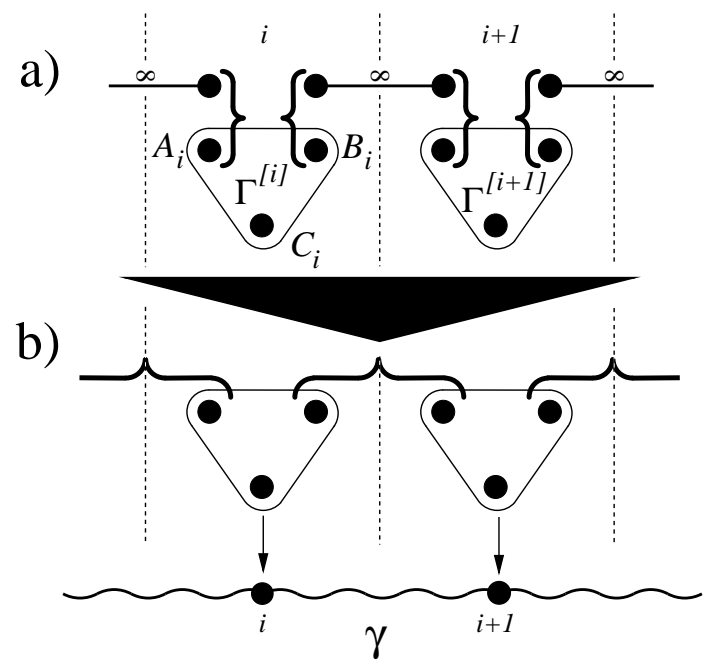

FIG. 6: If the local operations are described by states $\Gamma^{[i]}$ via the Jamiolkowski isomorphism, the construction of GMPS can be simplified by replacing the measurement-bond-measurement triples by a simple projection onto the EPR state. 
ments and one EPR pair can be replaced by a single projection onto the EPR state, Fig. 6 b. It follows that we can apply the formalism for projective measurements onto the EPR state which we presented in Sec. VIIA. We start from $\bigoplus_{i} \Gamma^{[i]}$. First we partially transpose all $A$ modes, then we collapse $A_{i+1}$ and $B_{i}$ for all $i$, and finally we take the Schur complement of the merged mode. In case of periodic boundary conditions, this can be expressed by the transformation matrix

$$
\Pi=\left(\begin{array}{ccc}
\mathbb{1}_{A} & \mathcal{R} \theta_{B} & 0 \\
0 & 0 & \mathbb{1}_{C}
\end{array}\right)
$$

which maps $A B C$ onto $A^{\prime} C$, where $\theta_{B} \equiv \theta \otimes \mathbb{1}$ is the partial transposition on system $B$, and $\mathcal{R}$ is the circulant right shift operator, $(\mathcal{R})_{i j}=\delta_{i, j+1} \bmod N \otimes \mathbb{1}$. Then, the output state, i.e., the GMPS characterized by $\Gamma^{[i]}$, is

$$
\gamma=\mathrm{SC}_{A^{\prime}}\left[\Pi\left(\bigoplus_{i} \Gamma^{[i]}\right) \Pi^{T}\right]
$$

where $\mathrm{SC}_{X}(U)$ is the Schur complement of the $X$ part of $U, \mathrm{SC}_{X}(U)=U_{Y Y}-U_{Y X} U_{X X}^{-1} U_{X Y}$. For fixed boundary conditions, the matrix $\Pi$ has to be modified accordingly at the boundaries. All the involved operations scale polynomially in the product $N M$ of the number of sites $N$ and the number of modes $M$.

In case all the local maps are chosen equal, $\Gamma^{[i]} \equiv \Gamma \forall i$, the above formula can be simplified considerably. Therefore, note that the Fourier transform can be taken into the Schur complement, and that $\Pi$ as well as $\bigoplus_{i=1}^{N} \Gamma^{[i]}=\Gamma \otimes \mathbb{1}_{N}$ are blockwise circulant so that both are diagonalized by the Fourier transform. We again adapt the notation of writing the diagonal of the Fourier transformed matrices as functions of an angle $\phi$, cf. Sec. III] In that case, $\Gamma \otimes \mathbb{1}$ is mapped onto the constant function $\Gamma$, and the same holds for $\mathbb{1}$ and $\theta$ in (48). The right shift operator $\mathcal{R}$, on the other hand, is transformed to $e^{i \phi} \mathbb{1}$ : the EPR measurement performed between adjacent sites leads to a complex phase of $\phi$. Altogether, we have

$$
\hat{\Pi}=\left(\begin{array}{ccc}
\mathbb{1}_{A} & e^{i \phi} \theta_{B} & 0 \\
0 & 0 & \mathbb{1}_{C}
\end{array}\right) ; \hat{\gamma}=\mathrm{SC}_{A^{\prime}}\left[\hat{\Pi} \Gamma \hat{\Pi}^{\dagger}\right]
$$

Directly expressed in terms of the map $\Gamma$, this reads

$$
\hat{\gamma}(\phi)=\Gamma_{C}-\Gamma_{C \mid A B} \hat{\Lambda} \frac{1}{\hat{\Lambda} \Gamma_{A B \mid A B} \hat{\Lambda}^{\dagger}} \hat{\Lambda}^{\dagger} \Gamma_{A B \mid C}
$$

where $\hat{\Lambda}=\left(\mathbb{1}_{A} ; e^{i \phi} \theta_{B}\right)$ is the upper left subblock of $\hat{\Pi}$.

\section{E. States with rational trigonometric functions as Fourier transforms}

If one restricts to pure MPS (i.e., those for which $\Gamma$ is pure) with one mode per site, then it follows from Theorem 2 that these states have reflection symmetry, and therefore $\hat{\gamma}(\phi)=\gamma_{0}+2 \sum_{n \geq 0} \gamma_{n} \cos (n \phi)$ is real. This implies that the sines in (49) can only appear in even powers $\sin ^{2 n} \phi=\left(1-\cos ^{2} \phi\right)^{n}$. Therefore, the Fourier transform $\hat{\gamma}$ of any pure Gaussian MPS, which is a $2 \times 2$ matrix valued function of $\phi$, has elements which are rational functons of $\cos (\phi),(\hat{\gamma}(\phi))_{x y}=p_{x y}(\cos (\phi)) / q_{x y}(\cos (\phi))$ with $p, q$ polynomials. The degree of the polynomials is limited by the size of $\hat{\Lambda} \Gamma_{A B} \hat{\Lambda}^{\dagger}$, and thus by the number $M$ of the bonds. One can easily check that $\operatorname{dim} p \leq 2 M+1$ and $\operatorname{dim} q \leq 2 M$.

For the following discussion, let us write those rational functions with a common denominator $d$,

$$
\hat{\gamma}(\phi)=\frac{1}{d(\cos (\phi))}\left(\begin{array}{cc}
q(\cos (\phi)) & r(\cos (\phi)) \\
r(\cos (\phi)) & p(\cos (\phi))
\end{array}\right)
$$


where $q, p, r$, and $d$ are polynomials of degree $L$. Then, the set of all such $\hat{\gamma}$ with $L \geq 2 M+1$ encompasses the set of translational invariant GMPS with $M$ bonds. Computing correlation functions in a lattice of size $N$ can be done straightforwardly in this representation by taking the discrete Fourier transform of $\hat{\gamma}(\phi)$ which scales polynomially with $N$, and in the following section we show that for one dimension, the correlations can be even computed exactly in the limit of an infinite chain.

It is interesting to note that $\gamma(\phi)$ is already determined up to a finite number of possibilities by fixing $r$ and $d$. Since $\gamma$ is pure, $1=\operatorname{det} \gamma=\operatorname{det} \hat{\gamma}$, and therefore, $p q=d^{2}+r^{2}$. Therefore, the zeros of $p q$ are the zeros of $d^{2}+r^{2}$, such that the only freedom is to choose how to distribute the zeros on $p$ and $q$. On the contrary, fixing only $q$ and $d$ does not give sufficient information, while choosing $p, q$ and $d$ (i.e., the diagonal of $\hat{\gamma}$ ) does not ensure that there exists a polynomial $r$ such that $p q-r^{2}=d^{2}$.

From the above, it follows that $2 L+1$ parameters are sufficient to describe $\hat{\gamma}(\phi)$, where $L$ is still the degree of the polynomials. This encloses all translational invariant Gaussian MPS with bond number $M \leq(L-1) / 2$, which need $(2 M+1)(2 M+2)=L(L+1)$ parameters. Therefore, the class of states where $\hat{\gamma}(\phi)$ is a rational function of $\cos (\phi)$ is a more efficient description of translationally invariant states than Gaussian MPS are.

Let us stress once more that the results of this section hold for arbitrary spatial dimension.

\section{F. Correlation length}

In the following, we show that the correlations of one-dimensional GMPS decay exponentially and explicitly derive the correlation length. The derivation only makes use of the representation (50) of Gaussian MPS and thus holds for the whole class of states where the Fourier transform is a rational function of the cosine. We will restrict to the case where the state $\Gamma$ associated to the GMPS map has only finite entries, which corresponds to the case where the denominator $d(\cos (\phi))$ in (50) has no zero on the unit circle. ${ }^{6}$

The correlations are directly obtained by back-transforming the elements of $\hat{\gamma}(\phi)$, which are rational functions $[\hat{\gamma}(\phi)]_{s}=s(\cos (\phi)) / d(\cos (\phi)), s \in\{p, q, r\}$; in the limit of an infinite chain,

$$
\left(\gamma_{s}\right)_{n}=\frac{1}{2 \pi} \int_{0}^{2 \pi} \frac{s(\cos (\phi))}{d(\cos (\phi))} e^{i n \phi} \mathrm{d} \phi
$$

Now transform $s, d$ to complex polynomials via $\cos (\phi) \rightarrow(z+1 / z) / 2$, and expand with $z^{K}$, $\tilde{s}(z):=z^{K} s(z), \tilde{d}(z):=z^{K} d(z)$, where $K$ is chosen large enough to make $\tilde{s}, \tilde{d}$ polynomials in $z$. Then,

$$
\begin{aligned}
\left(\gamma_{s}\right)_{n} & =\frac{1}{2 \pi i} \int_{\mathcal{S}^{1}} \frac{\tilde{s}(z) z^{n-1}}{\tilde{d}(z)} \mathrm{d} z \\
& =\sum_{z_{i}: \tilde{d}\left(z_{i}\right)=0} \frac{1}{\left(\nu_{i}-1\right) !} \underbrace{\left.\frac{\mathrm{d}^{\nu_{i}-1}}{\mathrm{~d} z^{\nu_{i}-1}}\left[\frac{\tilde{s}(z) z^{n-1}}{\tilde{d}_{i}(z)}\right]\right|_{z=z_{i}}}_{D_{i}}
\end{aligned}
$$

by the calculus of residues, where $\nu_{i}$ is the order of the zero $z_{i}$ in $\tilde{d}$ and $\tilde{d}_{i}(z)\left(z-z_{i}\right)^{\nu_{i}}=\tilde{d}(z)$. For $n>\nu_{i}, D_{i} \propto z_{i}^{\left(n-\nu_{i}\right)}$, and it follows that the correlations decay exponentially, where the correlation length is given by the largest zero of $q(z)$ inside the unit circle.

This proof holds only for one-dimensional GMPS. However, it can be proven for arbitrary spatial dimensions that the correlations decay as $o\left(\|n\|^{-\infty}\right)$ by iterated integration by parts as in Lemma 5 .

6 The case where $d$ has zeros on the unit circle corresponds to critical systems, which is why the correlations diverge. In the case of a Hamiltonian $H=V \oplus \mathbb{1}$, however, the ground state correlations of $P$ do not diverge. As in that case one has $p q=d^{2}, p / d=d / q$ need not have a singularity just because $q / d$ has one. 


\section{G. GMPS as ground states of local Hamiltonians}

Finally, we prove that every Gaussian MPS is the ground state of a local Hamiltonian, and show that only a special class of local Hamiltonians has a GMPS as an exact ground state. Again, the proof only relies on the representation (50).

Given a state $\gamma$ with Fourier transform (50), define $H$ to be the Hamiltonian matrix with Fourier transform

$$
\hat{H}(\phi)=\left(\begin{array}{cc}
p(\cos (\phi)) & -r(\cos (\phi)) \\
-r(\cos (\phi)) & q(\cos (\phi))
\end{array}\right) .
$$

Then, $H$ corresponds to a local Hamiltonian - the interaction range is the degree of $p, q, r-$ and according to (9), $\mathcal{E}(\phi)=\left[\sqrt{p q-r^{2}}\right](\cos \phi)=d(\cos \phi)$, which together with Eq. (10) proves that $\gamma$ is the ground state of $H$.

It is interesting to have a brief look at the converse as well. Given a local Hamiltonian, when will it have a GMPS as its ground state? Any local Hamiltonian has a Fourier transform which consists of polynomials in $\cos (\phi)$, and thus we adapt the notation of Eq. (51). Then, the ground state is represented by a rational function of $\cos (\phi)$ in Fourier space exactly if $p q-r^{2}=d^{2}$ is the square of another polynomial, as can be seen from Eq. (10). In terms of the original Hamiltonian, this implies that $H_{Q} H_{P}-H_{Q P}^{2}$ has to be the square of another banded matrix. For example, for the usual case $H=V \oplus \mathbb{1}$ one would need $V=X^{2}$ with $X$ again a banded matrix. The Klein-Gordon Hamiltonian (1), e.g., does not have a GMPS as its ground state.

\section{Acknowledgments}

We would like to thank Jens Eisert, Otfried Gühne, David Pérez García, Diego Porras, Tommaso Roscilde, Frank Verstraete, and Karl Gerd Vollbrecht for helpful discussions and comments. This work has been supported by the EU IST projects QUPRODIS and COVAQIAL.

\section{Appendix A: Hamiltonian simulation}

In this Appendix, we discuss the following question. Consider the set $\mathcal{S}$ of symplectic transformations of $N$ harmonic oscillators on a ring which have both translation and reflection symmetry. Assume that we can implement every local transformation of the form $S \oplus \ldots \oplus S \in \mathcal{S}$ and in addition one element of $\mathcal{S}$ corresponding to a nearest-neighbor interaction Hamiltonian. Is this set universal for simulating any operation in $\mathcal{S}$ ?

\section{Preliminaries}

Lie-Trotter formulae: It is most convenient to discuss the problem on the level of the Lie algebra of the symplectic group, since starting with a fixed set of operations in $\mathcal{S}$ the "reachable" transformations are characterized by the closure of the Lie algebra. That is, if $e^{\lambda A}$ and $e^{\lambda B}$ are reachable for every real $\lambda$ then so is $e^{\alpha A+\beta B}$ and $e^{\gamma[A, B]}$ for all $\alpha, \beta, \gamma \in \mathbb{R}$. This follows from the Lie-Trotter formulae

$$
\begin{aligned}
e^{\alpha A+\beta B} & =\lim _{n \rightarrow \infty}\left(e^{\alpha A / n} e^{\beta B / n}\right)^{n} \text { and } \\
e^{[A, B]} & =\lim _{n \rightarrow \infty}\left(e^{A / \sqrt{n}} e^{B / \sqrt{n}} e^{-A / \sqrt{n}} e^{-B / \sqrt{n}}\right)^{n} .
\end{aligned}
$$

The Lie algebra: Let $S=e^{t A}$ be a symplectic transformation close to the identity, i.e., $|t| \ll 1$. By examining the first order in $t$ we see that the condition $S \sigma S^{T}=\sigma$ is equivalent 
to $A \sigma=(A \sigma)^{T}$. Hence, we get all possible generators $A$ by multiplying any real symmetric matrix with the symplectic matrix $\sigma$ so that

$$
A=\left(\begin{array}{cc}
P & C \\
C^{T} & Q
\end{array}\right) \sigma^{T}=\left(\begin{array}{cc}
-C & P \\
-Q & C^{T}
\end{array}\right),
$$

where $Q, P$ are symmetric and the block structure corresponds to a direct sum of momentum and position space.

If $S \in \mathcal{S}$ then $Q, P$ and $C$ have to be circulant matrices (translation symmetry) and $C=C^{T}$ (reflection symmetry). Under these symmetries (and utilizing the fact that all circulant matrices mutually commute) the blocks of the commutator $A^{\prime \prime}=\left[A, A^{\prime}\right]$ are given by

$$
\begin{aligned}
& P^{\prime \prime}=2\left(C^{\prime} P-C P^{\prime}\right), \\
& Q^{\prime \prime}=2\left(C Q^{\prime}-C^{\prime} Q\right), \\
& C^{\prime \prime}=Q^{\prime} P-Q P^{\prime} .
\end{aligned}
$$

Note that, even if we do not assume reflection symmetry for $A$ and $A^{\prime}$, then $A^{\prime \prime}$ will have it, i.e., $C^{\prime \prime}$ will be symmetric. For this reason it is crucial to impose reflection symmetry otherwise the set of operations will not be universal.

For local transformations of the form $S \oplus \ldots \oplus S \in \mathcal{S}$ the blocks $P, Q$ and $C$ are proportional to the identity, since $\bigoplus_{i} e^{A_{i}}=e^{\oplus_{i} A_{i}}$.

Quadratic Hamiltonians: The relation between the Hamiltonian matrix of a quadratic Hamiltonian and the generator of the respective symplectic transformation can be obtained from the equation

$$
e^{i \mathcal{H} t} R_{n} e^{-i \mathcal{H} t} \equiv \sum_{l} S_{k l} R_{l}, \quad S=e^{A t} .
$$

Examining the infinitesimal regime yields

$$
A=\sigma H=\left(\begin{array}{cc}
H_{Q P}^{T} & H_{P} \\
-H_{Q} & -H_{Q P}
\end{array}\right) .
$$

Note that in general a symplectic transformation generated by an $A$ of the form in Eq. (A3) does not correspond to a semi-bounded Hamiltonian. However, by Eq. (A1) we can always decompose any symplectic transformation into time evolutions each of which is governed by a semi-definite Hamiltonian matrix. Moreover, if we can simulate the time evolution governed by a Hamiltonian $\mathcal{H}$ we can in principle also simulate the evolution according to $-\mathcal{H}$ by going to the revival time $T_{\text {rev }}$ for which

$$
e^{i \mathcal{H}\left(T_{\mathrm{rev}}-t\right)}=e^{-i \mathcal{H} t} .
$$

\section{Universality}

Theorem 16 Consider any transformation corresponding to a nearest-neighbor interaction Hamiltonian in the set $\mathcal{S}$ of translationally invariant symplectic operations with reflection symmetry. Together with all local transformations of the form $S \oplus \ldots \oplus S \in \mathcal{S}$ this is universal for simulating any transformation in $\mathcal{S}$.

Proof The theorem is proven in two steps. First we show that using additional local transformations only, we can extract each block in Eq. (A3) in the sense that one can simulate a new operation, where two of the three matrices $Q, P, C$ are set to zero, while the third remains unchanged. In this way we can in the second step discuss the reachability for each of these three components separately:

Extracting the blocks. - We will only show how the $P$-block can be extracted. Similar reasoning will then apply for the $Q$-block and by utilizing Eq. (A1) we can extract the $C$-components by simply subtracting the $Q$ and $P$ blocks. 
Let us start with a general $A$ of the form in Eq. (A3) and first get rid of the $C$-component. To this end consider the commutation relation (A4) with $C^{\prime}=\frac{1}{2} \mathbb{1}$ and $Q^{\prime}=P^{\prime}=0$. Then $P^{\prime \prime}=P, Q^{\prime \prime}=Q$ and $C^{\prime \prime}=0$. Starting with an $A$ of this form $(C=0)$ we may take the commutator with $Q^{\prime}=\frac{1}{2} \mathbf{1}, C^{\prime}=P^{\prime}=0$. Then $P^{\prime \prime}=Q^{\prime \prime}=0$ and $C^{\prime \prime}=P$. The commutator of such a matrix in turn with $P^{\prime}=-\frac{1}{2} \mathbb{1}, Q^{\prime}=C^{\prime}=0$ leads then finally to $P^{\prime \prime}=P$ with the other components zero.

Generating a basis.-Consider a matrix $A$ which corresponds to a nearest-neighbor interaction, i.e. in one of the blocks $Q, P, C$ the first off-diagonal is non-zero. By taking commutators like in (i) we can easily construct a second generator $A^{\prime}$, also corresponding to such an interaction, however with the respective off-diagonal in one of the other two blocks. By Eq. (A4) taking the commutator $\left[A, A^{\prime}\right]$ will lead to a product of the two non-diagonal circulant matrices in one of the blocks. This product will again be a circulant matrix, but now with a non-zero second off-diagonal. By iterating this procedure we can subsequently generate non-zero entries in every off-diagonal and taking linear combinations of these matrices will thus lead to a basis of symmetric circulant matrices in each of the blocks. Hence, together with (i) every generator corresponding to an element of $\mathcal{S}$ can be constructed.

\section{Remarks}

Revival time and efficiency: Making use of the revival time (A7) might be a severe handicap concerning the efficiency of the simulation. In particular $T_{\text {rev }}$ of the interaction Hamiltonian $H_{\text {int }}$ might grow exponentially with the number $N$ of modes.

In this case there are two ways to speed up the simulation: either one supplements the set of transformations with an additional interaction which is such that it provides an efficient simulation of $-H_{\text {int }}$, or one starts with a "good" $H_{\text {int }}$ for which this is possible from the very beginning. Examples for the latter are the Hamiltonians

$$
\begin{aligned}
\mathcal{H} & =\sum_{i=1}^{N} Q_{i}^{2}+P_{i}^{2}+\alpha\left(Q_{i} P_{i+1}+P_{i} Q_{i+1}\right), \\
\mathcal{H} & =\sum_{i=1}^{N}\left(Q_{i}+Q_{i+1}\right)^{2}+\left(P_{i}-P_{i+1}\right)^{2} .
\end{aligned}
$$

In both cases we can efficiently simulate the evolution according to $-\mathcal{H}$ by first applying the symplectic transformation $Q \mapsto P, P \mapsto-Q$ and then changing the sign of the diagonal in $H$ by local operations.

[1] M. B. Plenio, J. Eisert, J. Dreissig, and M. Cramer, Phys. Rev. Lett. 94, 060503 (2005), quant-ph/0405142.

[2] M. Cramer, J. Eisert, M. B. Plenio, and J. Dreissig, Phys.Rev. A 73, 012309 (2006), quant$\mathrm{ph} / 0505092$.

[3] M. M. Wolf, Phys. Rev. Lett. 96, 010404 (2006), quant-ph/0503219.

[4] K. Audenaert, J. Eisert, M. B. Plenio, and R. F. Werner, Phys. Rev. A 66, 042327 (2002).

[5] A. Botero and B. Reznik, Phys. Rev. A 70, 052329 (2004), quant-ph/0403233.

[6] M. Asoudeh and V. Karimipour, Phys. Rev. A 72, 0332339 (2005), quant-ph/0506022.

[7] M. B. Plenio and F. L. Semiao, New J. Phys. 7, 73 (2005), quant-ph/0407034.

[8] M. B. Plenio, J. Hartley, and J. Eisert, New J. Phys. 6, 36 (2004), quant-ph/0402004.

[9] J. Eisert, M. B. Plenio, S. Bose, and J. Hartley, Phys. Rev. Lett. 93, 190402 (2004), quant$\mathrm{ph} / 0311113$

[10] M. M. Wolf, F. Verstraete, and J. Cirac, Int. J. Quant. Inf. 1, 465 (2003), quant-ph/0311051.

[11] M. M. Wolf, F. Verstraete, and J. Cirac, Phys. Rev. Lett. 92, 087903 (2004), quant-ph/0307060.

[12] B. Nachtergaele and R. Sims, Commun. Math. Phys. 265, 119 (2006), math-ph/0506030.

[13] M. B. Hastings and T. Koma, Commun.Math.Phys 265, 781 (2006), math-ph/0507008.

[14] M. Cramer and J. Eisert, New J. Phys. 8, 71 (2006), quant-ph/0509167.

[15] A. Auerbach, Interacting electrons and quantum magnetism (Springer Verlag, New York, 1994). 
[16] D. James, Appl. Phys. B 66, 181 (1998), quant-ph/9702053.

[17] J. Williamson, American Journal of Mathematics 58, 141 (1936).

[18] G. Birkl, S. Kassner, and H. Walther, Nature 357, 310 (1992).

[19] D. H. E. Dubin, Phys. Rev. Lett. 71, 2753 (1993).

[20] D. G. Enzer, M. M. Schauer, J. J. Gomez, M. S. Gulley, and et al., Phys. Rev. Lett. 85, 2466 (2000).

[21] T. B. Mitchell, J. J. Bollinger, D. H. E. Dubin, X.-P. Huang, W. M. Itano, and R. H. Baugham, Science 282, 1290 (1998).

[22] J. Manuceau and A. Verbeure, Comm. Math. Phys. 9, 293 (1968).

[23] A. S. Holevo, Theor. Math. Phys. 6, 1 (1971).

[24] Arvind, B. Dutta, N. Mukunda, and R. Simon, Pramana 45, 471 (1995), quant-ph/9509002.

[25] M. M. Wolf, G. Giedke, O. Krüger, R. F. Werner, and J. I. Cirac, Phys. Rev. A 69, 052320 (2004), quant-ph/0306177.

[26] M. Benzi and G. H. Golub, BIT Numerical Mathematics 39, 417 (1999).

[27] N. Bleistein and R. A. Handelsman, Asymptotic expansions of integrals (Dover Publication, New York, 1986).

[28] F. Verstraete, J. J. Garcia-Ripoll, and J. I. Cirac, Phys. Rev. Lett. 93, 207204 (2004), condmat/0406426.

[29] M. Zwolak and G. Vidal, Phys. Rev. Lett. 93, 207205 (2004), cond-mat/0406440.

[30] F. Verstraete and J. I. Cirac (2004), cond-mat/0407066.

[31] F. Verstraete, D. Porras, and J. I. Cirac, Phys. Rev. Lett. 93, 227205 (2004), condmat/0404706.

[32] F. Verstraete and J. Cirac, Phys. Rev. B 73, 094423 (2006), cond-mat/0505140.

[33] F. Verstraete, J. Cirac, J. Latorre, E. Rico, and M. Wolf, Phys. Rev. Lett. 94, 140601 (2005), quant-ph/0410227.

[34] C. Schön, E. Solano, F. Verstraete, J. I. Cirac, and M. M. Wolf, Phys. Rev. Lett. 05, 110503 (2005), quant-ph/0501096.

[35] A. Jamiołkowski, Rep. Math. Phys. 3, 275 (1972).

[36] G. Giedke and J. I. Cirac, Phys. Rev. A 66, 032316 (2002), quant-ph/0204085.

[37] J. Fiurášek, Phys. Rev. Lett. 89, 137904 (2002), quant-ph/0204069.

[38] A. S. Holevo, Probabilistic and statistical aspects of quantum theory (North-Holland Publishing Company, 1982).

[39] A. S. Holevo and R. F. Werner, Phys. Rev. A 63, 032312 (2001), quant-ph/9912067. 